\title{
ESC Abstract Awards: Highlights from the 23rd European Stroke Conference, Nice, May 6-9, 2014
}

\author{
M.G. Hennerici \\ Department of Neurology, UMM, University of Heidelberg, Mannheim, Germany
}

Nice hosted the European Stroke Conference (ESC) for the second time since 2008. The ESC 2014 took place at the newly refurbished Nice Acropolis Convention Centre located in the heart of the city and was attended by 3,632 participants including 204 invited speakers, chairpersons and committee members.

An exciting and outstanding programme was prepared, with teaching courses (attended by 1,045), the Nurses and AHP Educational Programme, more than 10 mini-symposia, joint society sessions and educational symposia as well as a large number of individual original scientific contributions: in a rigorous peer review process more than 1,500 abstracts of excellent quality had been submitted from 63 countries all over the world, including more than $40 \%$ from non-European countries. There were 286 oral communications, and for the first time 933 e-posters were presented using 30 electronic terminals, which was highly appreciated by presenters and attendants during two big guided poster sessions. This new technology also allowed participants to visualise posters through free access during the full meeting. A new interactive e-book including all abstracts finally accepted was available with a huge selection of tables and figures online during the conference and thereafter in our corresponding high impact-listed journal (IF 3.7) Cerebrovascular Diseases (http://misc.karger.com/products/ CED_2014_037_S1/index.html).

As usual, large clinical trial sessions were well attended, although to some extent results were disappointing: in contrast to the large number selected for two sessions $(\mathrm{n}=17)$, only 2 authors really presented new and important studies.

The initial presentation in the first session was given by Pierre Amarenco on behalf of the TIA Registry doc.org Investigators: he reported exciting data on the 1-year out- come of 4,789 patients included in their registry, which nicely demonstrated that modern management and urgent/emergency secondary stroke prevention strategies resulted in a considerably lower residual risk for stroke recurrence than previously reported. Furthermore, risk stratification is highly recommended taking into account that high $\mathrm{ABCD} 2$ scores and/or abnormal MRI findings, or large artery atherosclerosis as the most important subtype of stroke, all indicate higher stroke recurrence rates and profit from best medical treatment. The second important study was presented by H.C. Diener on behalf of the CRYSTAL AF Investigators in the last session of the meeting. He reported data on 441 patients randomised to insertable cardiac monitors versus standard care within 90 days after cryptogenic stroke. Atrial fibrillation was significantly more frequently detected in insertable cardiac monitor patients at 6 months ( 8.9 vs. $1.4 \%)$ and 12 months (12.4 vs. $2 \%)$ and led to a higher rate of oral anticoagulant prescription than was expected from the detection of atrial fibrillation before randomisation.

For the first time we introduced two Highlight sessions which extracted 'Take Home Messages' from 20 awarded research groups covering the full range from basic science to rehabilitation and prevention. We invited authors to submit extended, illustrated versions of their abstracts to Cerebrovascular Diseases, and 18 of them are published in this article. We hope that this is of major interest to our readers and also stimulates the motivation of investigators active in stroke research of whatever specific topic to contribute to future ESCs.

Michael G. Hennerici

Chairman of the ESC Programme Committee Editor of Cerebrovascular Diseases 


\section{Population-Based Studies}

\section{1 \\ Cortical Superficial Siderosis and Intracerebral Haemorrhage Risk in Patients with Sporadic Cerebral Amyloid Angiopathy: Multicentre MRI Cohort Study}

A. Charidimou ${ }^{a}$, A. Peeters ${ }^{e}$, H.R. Jäger ${ }^{b, c}$, , Z. Fox ${ }^{d}$, Y. Vandermeeren ${ }^{f, g}$, P. Laloux ${ }^{f, g}$, J.-C. Baron ${ }^{\text {h, } i}$, D.J. Werring ${ }^{a}$

aStroke Research Group, Department of Brain Repair and Rehabilitation, and ' Lysholm Department of Neuroradiology, UCL Institute of Neurology and National Hospital for Neurology and Neurosurgery, 'Department of Brain Repair and Rehabilitation, and ${ }^{\mathrm{d} B i o m e d i c a l}$ Research Centre, UCL and Education Unit, UCL Institute of Neurology, London, UK; eDepartment of Neurology, Cliniques Universitaires UCL St. Luc, Brussels, fDepartment of Neurology, CHU Mont-Godinne, Université Catholique de Louvain, Yvoir, and Institute of Neuroscience, Université Catholique de Louvain, Brussels, Belgium; ' Department of Clinical Neurosciences, University of Cambridge, Addenbrooke's Hospital, Cambridge, UK; 'UMR 894 INSERM-Université Paris 5, Sorbonne Paris Cité, Paris, France

\section{Background}

Sporadic cerebral amyloid angiopathy (CAA) is a highly prevalent, yet under-recognised, age-related disease [1]. It is caused by progressive amyloid- $\beta$ deposition in small cortical and leptomeningeal vessels. In some patients with severe disease, amyloid- $\beta$ deposits might cause the blood vessels to become brittle and fragile, so that blood can leak out leading to devastating lobar intracerebral haemorrhage $(\mathrm{ICH})$, the commonest recognised manifestation of CAA [1-4]. Lobar ICH associated with CAA carries a high risk for recurrence, which is probably even greater compared to deep ICH (presumed due to hypertensive arteriopathy) $[5,6]$, but this risk is currently difficult to predict.

Several factors predisposing to an increased lobar ICH risk have been described in CAA, including: apolipoprotein $\mathrm{E} \varepsilon 4$ and $\varepsilon 2$ alleles [7]; haemorrhagic neuroimaging markers of CAA such as lobar cerebral microbleeds on blood-sensitive MRI sequences [8], the number of haemorrhages (macro- or micro-) at baseline [8] and the use of antiplatelet treatment following symptomatic lobar ICH [9]. However, little is known about cortical superficial siderosis (cSS), a recently identified but common neuroimaging marker of CAA, and the risk of subsequent ICH. In CAA, cSS quite characteristically affects the cerebral convexities. It reflects blood breakdown products that line the outermost surface of the cortex or are in the subarachnoid space $[10,11]$. One likely underlying mechanism leading to cSS is repeated episodes of haemorrhage into the subarachnoid space from brittle superficial cortical or leptomeningeal CAA-laden vessels, potentially heralding a high risk of future lobar ICH [12].

We therefore investigated the hypothesis that cSS on MRI, especially cSS involving multiple sulci (i.e. disseminated, reflecting more widespread or active disease), is associated with an increased risk of future symptomatic ICH in patients with CAA.

\section{Methods}

We undertook a European multicentre retrospective cohort study of prospectively identified consecutive patients diagnosed with probable/possible CAA according to the Boston criteria [13] with available MRI and adequate follow-up. The centres were: UCL (University College London) Hospitals NHS Foundation Trust (London) (03/2003-09/2011), Addenbrooke's Hospital (Cambridge) (07/2002-03/2010), Cliniques Universitaires St. Luc (Brussels) (12/2003-04/2010) and CHU Mont-Godinne UCL (08/2005-03/2009). Standardized baseline clinical and imaging (MRI) data were collected. Follow-up data on symptomatic spontaneous lobar ICH were obtained from a systematic review of prospective databases, and medical and radiological records. Outcome events were assessed using all clinical, radiological and pathological information available, blinded to the presence of cSS at baseline MRI. cSS was defined as linear residues of chronic blood products in the superficial layers of the cerebral cortex showing a characteristic 'gyriform' pattern of low signal on T2*-GRE images, without corresponding hyperintense signal on T1-weighted or FLAIR images (i.e. without acute subarachnoid haemorrhage). We did not include cSS contiguous with any ICH. The distribution of cSS was classified as focal (restricted to $\leq 3$ sulci) or disseminated ( $>3$ sulci) [10]. Kaplan-Meier and Cox regression analyses were used to investigate cSS [focal or disseminated (i.e. $>3$ sulci involved)] as a predictor of future ICH risk, adjusting for known confounders, including the burden of cerebral microbleeds, age and previous history of symptomatic lobar ICH (fig. 1). 
Fig. 1. Kaplan-Meier estimates of progression to symptomatic lobar ICH in the presence of cSS (a), and focal or disseminated (>3 sulci) cSS $(\mathbf{b})$ in all patients with CAA. Testing of significance is by the log-rank test.

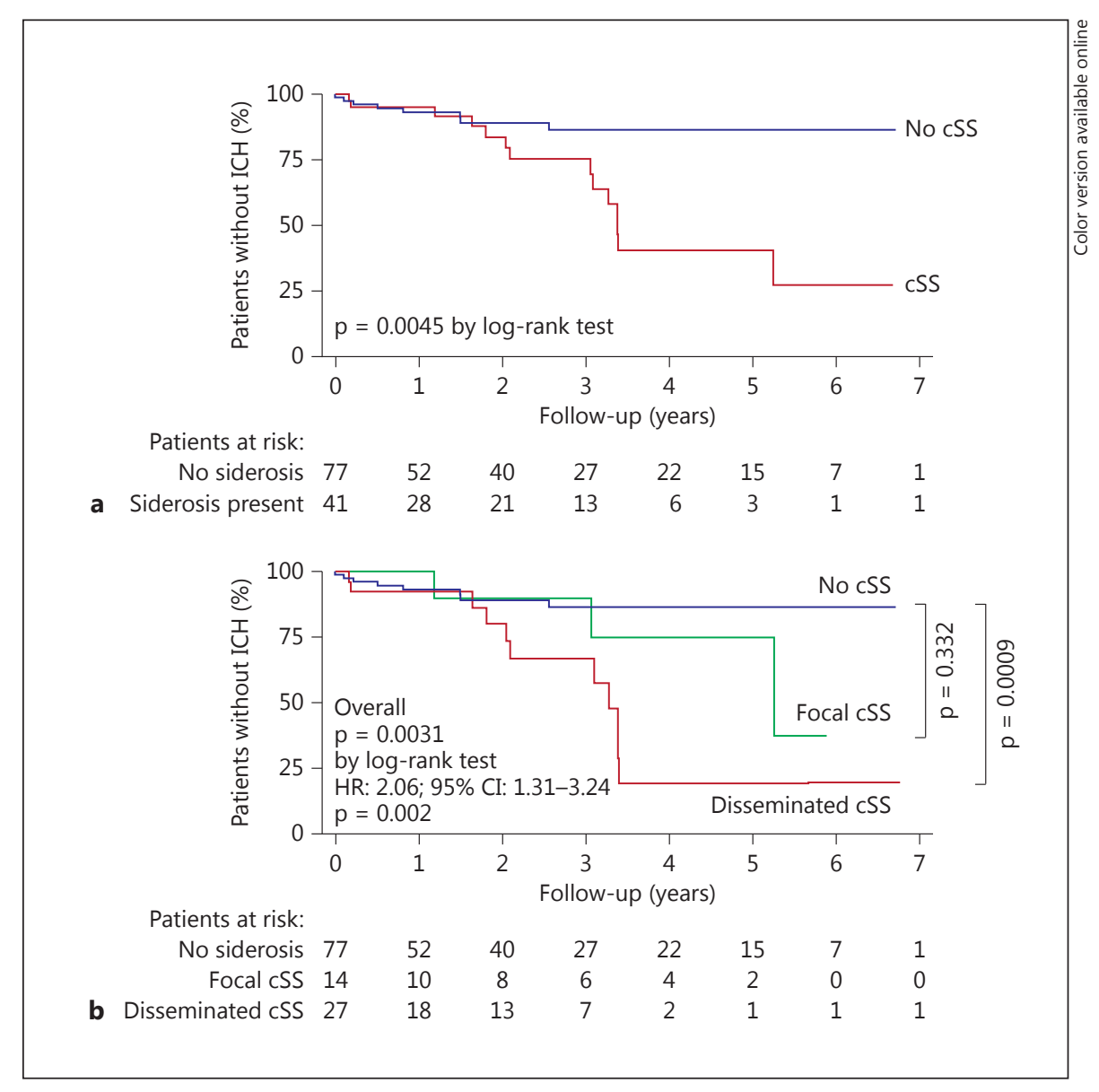

\section{Results}

The final cohort consisted of 118 patients fulfilling the diagnostic criteria for CAA: 8 patients with pathologically proven CAA, 86 patients with probable CAA and 24 patients with possible CAA based on the Boston criteria [13]. Of these, 104 (88\%) patients presented with symptomatic lobar ICH at baseline. The median time between all baseline symptomatic ICH and first MRI was 17 days (interquartile range, IQR: 3-75.5 days). Forty-one patients (43.8\%, 95\% CI: $26.2-44.6 \%)$ had cSS at baseline. During a median follow-up time of 24 months (IQR: 9-44 months), 23/118 (19.5\%; 95\% CI: 12.8-27.8) patients experienced symptomatic lobar ICH (table 1). Any cSS and disseminated cSS were predictors of time until first/recurrent ICH (log-rank test: $\mathrm{p}=0.0045$ and $\mathrm{p}=0.0009$, respectively). ICH risk at 4 years was $25 \%$ (95\% CI: $7.6-$ $28.3 \%$ ) for patients without siderosis; $28.9 \%$ (95\% CI: 7.7$76.7 \%$ ) for patients with focal siderosis, and $74 \%$ (95\% CI: 44.1-95.7) for patients with disseminated cSS (log-rank test: $p=0.0031)$. Any cSS and disseminated cSS were independently associated with increased lobar ICH risk after adjusting for $\geq 2$ microbleeds and age in Cox regression models (hazard ratio, HR: 2.53, 95\% CI: 1.05-6.15, $\mathrm{p}=0.040$, and HR: $3.16,95 \%$ CI: $1.35-7.43, \mathrm{p}=0.008$, respectively). Our main results were also consistent in sensitivity analyses including cSS, cerebral microbleeds, previous history of symptomatic ICH and age in multivariable models. These results also remained consistent in subgroup analyses of only patients with lobar ICH at baseline.

\section{Conclusions}

We have shown that cSS is associated with a high risk of ICH recurrence in patients diagnosed with CAA; the risk is particularly high if siderosis involves multiple cerebral sulci. Our findings suggest that cSS is a useful new prognostic marker in CAA and may help stratify future 
Table 1. Characteristics and comparison of CAA patients according to symptomatic lobar ICH at follow-up

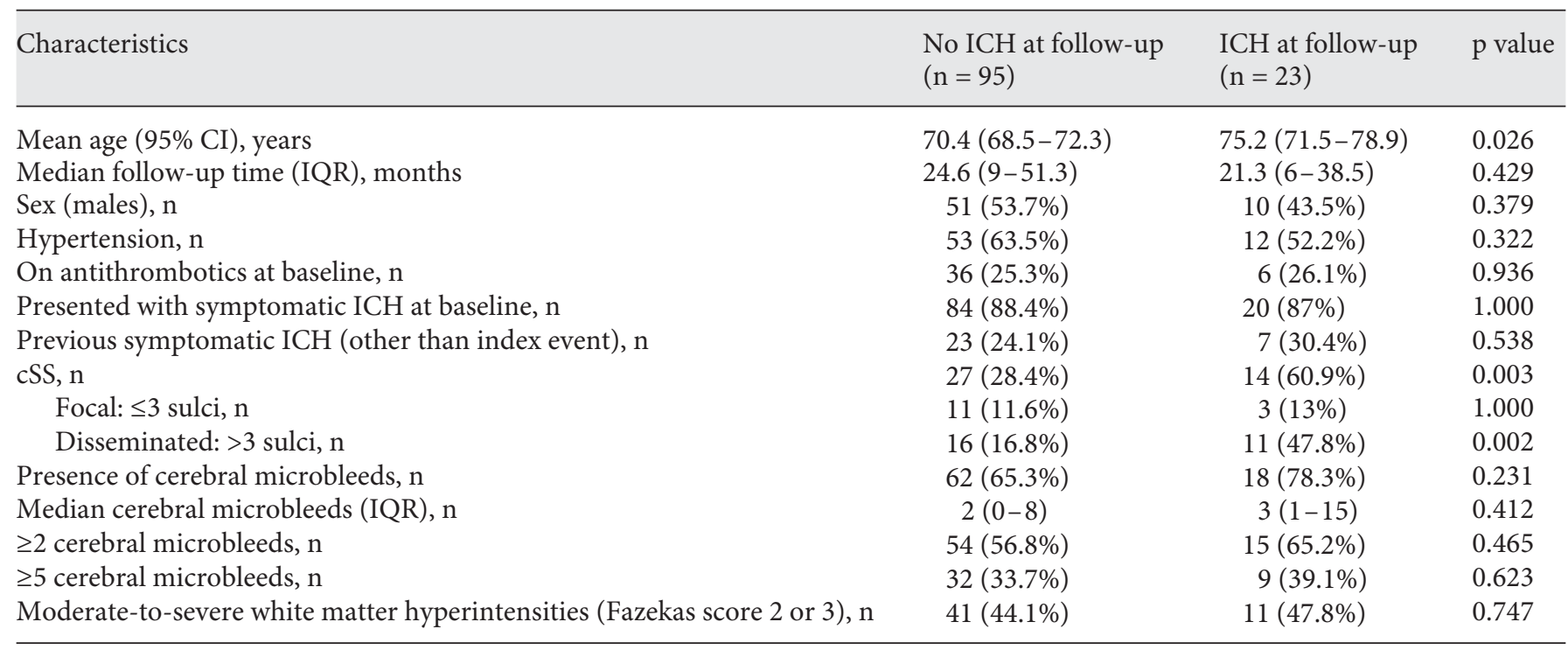

bleeding risk with implications for prognosis and treatment (e.g. avoiding antithrombotic agents in patients with disseminated cSS) or future clinical intervention studies. Larger cohorts are needed to confirm our results and further explore clinical implications.

\section{References}

1 Charidimou A, Gang Q, Werring DJ: Sporadic cerebral amyloid angiopathy revisited: recent insights into pathophysiology and clinical spectrum. J Neurol Neurosurg Psychiatry 2012;83:124-137.

2 Viswanathan A, Greenberg SM: Cerebral amyloid angiopathy in the elderly. Ann Neurol 2011;70:871-880.

3 Vinters HV: Cerebral amyloid angiopathy. A critical review. Stroke 1987; 18:311-324.

4 Bejot Y, Cordonnier C, Durier J, Aboa-Eboule C, Rouaud O, Giroud M: Intracerebral haemorrhage profiles are changing: results from the Dijon population-based study. Brain 2013;136(pt 2):658-664.

$\checkmark 5$ Bailey RD, Hart RG, Benavente O, Pearce LA: Recurrent brain hemorrhage is more frequent than ischemic stroke after intracranial hemorrhage. Neurology 2001;56:773-777.

-6 Viswanathan A, Rakich SM, Engel C, Snider R, Rosand J, Greenberg SM, Smith EE: Antiplatelet use after intracerebral hemorrhage. Neurology 2006;66:206-209.
7 O’Donnell HC, Rosand J, Knudsen KA, Furie KL, Segal AZ, Chiu RI, Ikeda D, Greenberg SM: Apolipoprotein E genotype and the risk of recurrent lobar intracerebral hemorrhage. N Engl J Med 2000;342:240245.

8 Greenberg SM, Eng JA, Ning M, Smith EE, Rosand J: Hemorrhage burden predicts recurrent intracerebral hemorrhage after lobar hemorrhage. Stroke 2004;35:1415-1420.

-9 Biffi A, Halpin A, Towfighi A, Gilson A, Busl K, Rost N, Smith EE, Greenberg MS, Rosand J, Viswanathan A: Aspirin and recurrent intracerebral hemorrhage in cerebral amyloid angiopathy. Neurology 2010;75:693698.

$\checkmark 10$ Linn J, Halpin A, Demaerel P, Ruhland J, Giese AD, Dichgans M, van Buchem MA, Bruckmann H, Greenberg SM: Prevalence of superficial siderosis in patients with cerebral amyloid angiopathy. Neurology 2010; 74:1346-1350.

11 Linn J, Herms J, Dichgans M, Bruckmann H, Fesl G, Freilinger T, Wiesmann M: Subarachnoid hemosiderosis and superficial cortical hemosiderosis in cerebral amyloid angiopathy. AJNR Am J Neuroradiol 2008;29: 184-186.

12 Linn J, Wollenweber FA, Lummel N, Bochmann K, Pfefferkorn T, Gschwendtner A, Bruckmann H, Dichgans M, Opherk C: Superficial siderosis is a warning sign for future intracranial hemorrhage. J Neurol 2013;260:176-181.

13 Knudsen KA, Rosand J, Karluk D, Greenberg SM: Clinical diagnosis of cerebral amyloid angiopathy: validation of the Boston criteria. Neurology 2001;56:537-539. 
Table 1. Carotid IMT in adulthood according to exposure to parental smoking in childhood or adolescence in the pooled dataset containing data from the YFS and CDAH study

\begin{tabular}{|c|c|c|c|c|c|c|c|c|c|c|c|c|c|}
\hline Parental smoking & $\mathrm{n}$ & \multicolumn{3}{|c|}{ Adjusted model $1^{\mathrm{a}}$} & \multicolumn{3}{|c|}{ Adjusted model $2^{\mathrm{b}}$} & \multicolumn{3}{|c|}{ Adjusted model $3^{c}$} & \multicolumn{3}{|c|}{ Adjusted model $4^{\mathrm{d}}$} \\
\hline Either parent smoked & 1,112 & 0.638 & 0.003 & 0.729 & 0.637 & 0.003 & 0.910 & 0.637 & 0.003 & 0.967 & 0.638 & 0.003 & 0.921 \\
\hline Both parents smoked & 408 & 0.653 & 0.004 & 0.001 & 0.653 & 0.004 & 0.001 & 0.652 & 0.005 & 0.003 & 0.652 & 0.005 & 0.003 \\
\hline$p_{\text {trend }}$ & & & & 0.005 & & & 0.011 & & & 0.021 & & & 0.017 \\
\hline
\end{tabular}

Values are estimated marginal means of maximum carotid IMT.

a Adjusted for age, sex and cohort. ${ }^{\mathrm{b}}$ Adjusted for age, sex, cohort, parental education, childhood body mass index (BMI) and childhood smoking. ${ }^{c}$ Adjusted for age, sex, cohort, parental educa- tion, childhood BMI, childhood smoking, adult education and adult smoking status. ${ }^{\mathrm{d}}$ Adjusted for age, sex, cohort, parental education, childhood BMI, childhood smoking, adult education and adult smoking status, adult systolic blood pressure, adult LDL cholesterol, adult triglycerides and adult C-reactive protein.
2

Exposure to Parental Smoking in Childhood Is Associated with Increased Carotid Intima-Media Thickness in Young Adults: Evidence from Two Prospective Cohort Studies in Australia and Finland

S. Galla, Q.L. Huynh', C.G. Magnussen ${ }^{a}$, M. Juonalac, J.S.A. Viikaric, M. Kähönen ${ }^{d}$, T. Dwyer ${ }^{b}$, O.T. Raitakaric, A. Venn ${ }^{a}$

aUniversity of Tasmania, Menzies Research Institute Tasmania, Hobart, Tas., and burdoch Childrens Research Institute, Melbourne, Vic., Australia; 'University of Turku, Turku, and dUniversity of Tampere, Tampere, Finland

\section{Background}

It is estimated that 700 million children are exposed to passive smoking worldwide [1]. It is, therefore, surprising that we know very little about the long-term cardiovascular effects of smoking exposure, with no longitudinal studies examining whether exposure to parental smoking in childhood adversely affects the vascular structure into adulthood. We examined this association in two cohorts followed up to 25 years from childhood to adulthood, with results recently published in the European Heart Journal [2].

\section{Methods}

The study comprised participants from the Cardiovascular Risk in Young Finns Study (YFS, $n=2,401$ ) and the Childhood Determinants of Adult Health $(\mathrm{CDAH}, \mathrm{n}=$ 1,375 ) study. Exposure to parental smoking (none, one or both), total number of smokers in the home (CDAH study only, range: $0-5$ ) and cumulative exposure to parental smoking over a 3-year period [YFS only, range: 0 (no exposure 1983 and 1986) to 4 (both parents 1983 and 1986)] was assessed by questionnaire. B-mode ultrasound of the carotid artery determined intima-media thickness (IMT) in adulthood using a common protocol. Linear regression on a pooled dataset accounting for the hierarchical data and potential confounders, including age, sex, parental education, participant smoking, education and adult cardiovascular risk factors, was conducted.

\section{Results}

Carotid IMT in adulthood was greater in those exposed to both parents' smoking than those whose parents did not smoke (table 1 , adjusted marginal means \pm SE: $0.647 \pm 0.022$ vs. $0.632 \pm 0.021 \mathrm{~mm}, \mathrm{p}=0.004)$. The effect was independent of participant smoking at baseline and follow-up and other confounders, including adult cardiovascular risk factors, and was uniform across categories of age, sex, adult smoking status and cohort. It equated to a vascular age 3.3 years greater in those exposed compared to those not exposed. In $\mathrm{CDAH}$, each additional person smoking in the home was associated with a 0.007 $\pm 0.003 \mathrm{~mm}(\mathrm{p}=0.025)$ increase in IMT in adulthood (table 2), while in the YFS greater cumulative exposure from 1980 to 1983 was associated with greater IMT in adulthood (per unit of exposure $0.003 \pm 0.002 \mathrm{~mm}, \mathrm{p}=$ 0.066 ). Sensitivity analyses examined whether adjusting for alternative measures of covariates available in a re- 
Table 2. Difference in carotid IMT per unit increase in exposure to parental smoking in childhood or adolescence in the CDAH study and YFS

\begin{tabular}{|c|c|c|}
\hline & $\begin{array}{l}\text { CDAH } \\
(\mathrm{n}=1,362 \text { smokers } \\
\text { at home/smoker })\end{array}$ & $\begin{array}{l}\text { YFS } \\
(\mathrm{n}=2,041 \text { cumulative } \\
\text { parental smoking } \\
\text { exposures/ unit) }{ }^{\mathrm{a}}\end{array}$ \\
\hline \multicolumn{3}{|l|}{ Adjusted model $1^{\mathrm{b}}$} \\
\hline$\beta \pm S E$ & $0.007 \pm 0.003$ & $0.004 \pm 0.002$ \\
\hline $\mathrm{p}$ & 0.021 & 0.034 \\
\hline \multicolumn{3}{|c|}{ Adjusted model $2^{\mathrm{c}}$} \\
\hline$\beta \pm S E$ & $0.007 \pm 0.003$ & $0.003 \pm 0.002$ \\
\hline $\mathrm{p}$ & 0.013 & 0.060 \\
\hline \multicolumn{3}{|c|}{ Adjusted model $3^{\text {d }}$} \\
\hline$\beta \pm S E$ & $0.007 \pm 0.003$ & $0.003 \pm 0.002$ \\
\hline $\mathrm{p}$ & 0.025 & 0.066 \\
\hline \multicolumn{3}{|c|}{ Adjusted model $4^{\mathrm{e}}$} \\
\hline$\beta \pm S E$ & $0.007 \pm 0.003$ & $0.003 \pm 0.002$ \\
\hline $\mathrm{p}$ & 0.022 & 0.083 \\
\hline \multicolumn{3}{|c|}{$\begin{array}{l}\text { a Range: } 0 \text { (no parents smoked at either time point) to } 4 \text { (both } \\
\text { parents smoked at both time points). } \\
\text { b Adjusted for age and sex. } \\
{ }^{c} \text { Adjusted for age, sex and parental education. } \\
\text { d Adjusted for age, sex, parental education, adult education and } \\
\text { adult smoking status. } \\
\text { e Adjusted for age, sex, parental education, adult education, } \\
\text { adult smoking status, adult body mass index, adult LDL choleste- } \\
\text { rol and C-reactive protein. }\end{array}$} \\
\hline
\end{tabular}

duced sample affected the results. These included childhood socioeconomic status or smoking and adult packyears of smoking or passive smoking (nonsmokers in YFS only). The results were unchanged with adjustment for these alternative covariates.

\section{Conclusions}

These results suggest a direct and pervasive effect of exposure to parental smoking on children's vascular health up to 25 years later. It is, therefore, imperative that we continue efforts to reduce smoking among adults to protect children and reduce the burden of cardiovascular disease across the population.

\section{References}

1 World Health Organization: International Consultation on Environmental Tobacco Smoke (ETS) and Child Health. Geneva, WHO, 1999.
Gall S, Huynh QL, Magnussen CG, Juonala M, Viikari JS, Kahonen M, et al: Exposure to parental smoking in childhood or adolescence is associated with increased carotid intima-media thickness in young adults: evidence from the Cardiovascular Risk in Young Finns study and the Childhood Determinants of Adult Health Study. Eur Heart J 2014, Epub ahead of print.

3

Copeptin and Long-Term Risk of Recurrent Vascular Events after Transient Ischaemic Attack and Ischaemic Stroke: A Population-Based Study

S. Greisenegger, H. Segal, A. Burgess, D. Poole, Z. Mehta, P.M. Rothwell

Stroke Prevention Research Unit, Nuffield Department of Clinical Neurosciences, University of Oxford, Oxford, UK

\section{Background}

Copeptin, the C-terminal-portion of pro-vasopressin, is a useful prognostic marker in patients after myocardial infarction (MI) and heart failure. More recently, high levels of copeptin have also been associated with worse functional outcome and increased mortality 3 months after ischaemic stroke and transient ischaemic attack (TIA). However, to date, there are no published data on the long-term prognostic value of copeptin after TIA or stroke.

\section{Methods}

We analysed copeptin in consecutive eligible patients with TIA or minor ischaemic stroke in a populationbased study (Oxford Vascular Study) recruited from 2002 and followed up to 2013. Levels were measured with a sandwich immunoluminometric assay as soon as possible after the index event and after 1 year. Associations with the risk of recurrent vascular events were determined by Cox regression.

\section{Results}

Copeptin levels were determined in 839 patients at baseline (median time to sampling 5 days, interquartile range: 2-10) and in 309 patients after 1 year. During about 5,000 patient-years of follow-up, with a median of 6.4 years, there were 131 recurrent strokes, $46 \mathrm{MI}$ and 131 
Table 1. Associations of copeptin and recurrent vascular events and death

\begin{tabular}{|c|c|c|c|c|c|c|c|c|}
\hline & \multicolumn{2}{|c|}{$\begin{array}{l}\text { Recurrent ischaemic } \\
\text { stroke/MI (177 events) }\end{array}$} & \multicolumn{2}{|c|}{$\begin{array}{l}\text { Recurrent ischaemic } \\
\text { stroke (131 events) }\end{array}$} & \multicolumn{2}{|l|}{$\begin{array}{l}\text { Vascular death } \\
\text { (131 events) }\end{array}$} & \multicolumn{2}{|l|}{$\begin{array}{l}\text { All-cause death } \\
\text { (317 events) }\end{array}$} \\
\hline & HR (95\% CI) & $p$ value & HR (95\% CI) & $\mathrm{p}$ value & HR (95\% CI) & $\mathrm{p}$ value & HR (95\% CI) & $\mathrm{p}$ value \\
\hline Unadjusted & $1.50(1.31-1.73)$ & $<0.0001$ & $1.42(1.21-1.68)$ & $<0.0001$ & $1.97(1.68-2.31)$ & $<0.0001$ & $1.75(1.58-1.95)$ & $<0.0001$ \\
\hline Model 1 & $1.42(1.22-1.64)$ & $<0.0001$ & $1.37(1.15-1.62)$ & 0.0004 & $1.76(1.49-2.08)$ & $<0.0001$ & $1.56(1.39-1.74)$ & $<0.0001$ \\
\hline Model 2 & $1.28(1.09-1.50)$ & 0.0028 & $1.31(1.10-1.58)$ & 0.004 & $1.59(1.33-1.91)$ & $<0.0001$ & $1.51(1.34-1.71)$ & $<0.0001$ \\
\hline
\end{tabular}

Model 1: Adjusted for age and sex. Model 2: model 1 plus adjustment for hypertension, diabetes, previous MI, previous stroke, previous peripheral vascular disease, current smoker, chronic heart failure, atrial fibrillation, previous antiplatelet agents, previous antihypertensive therapy and previous statins.

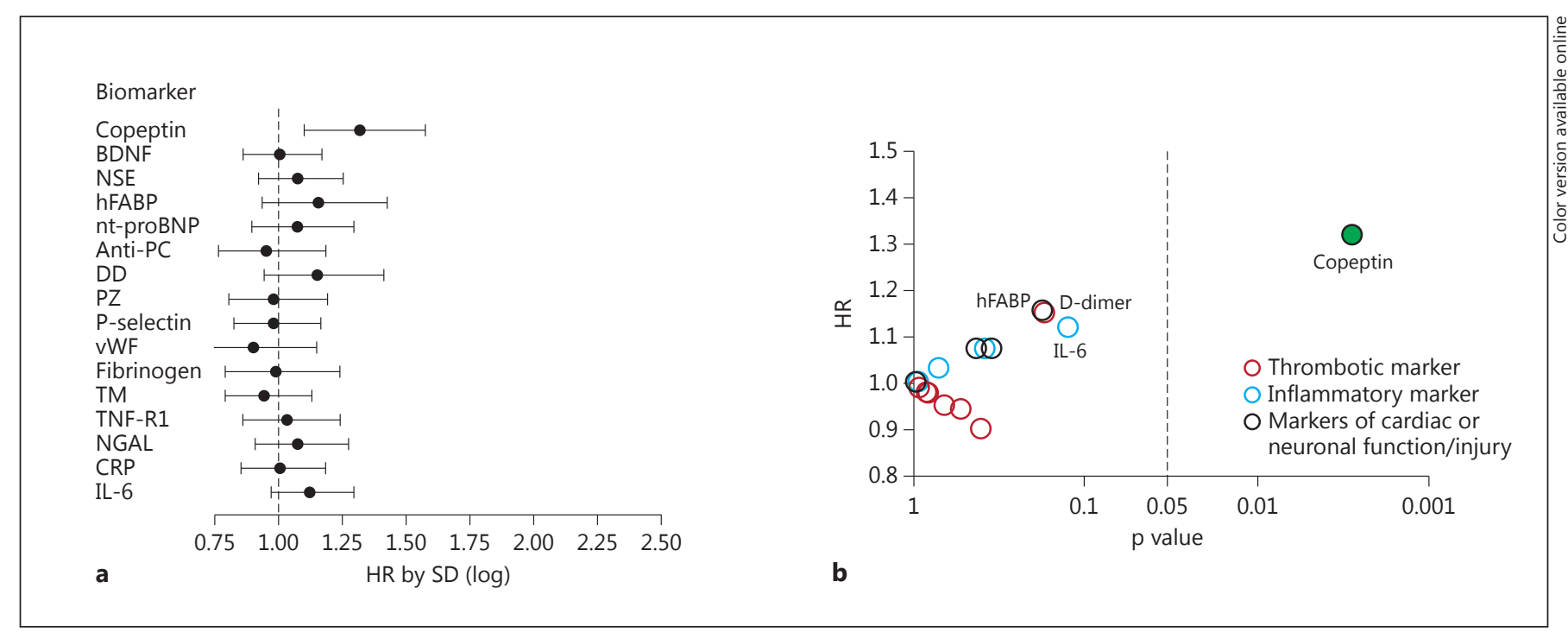

Fig. 1. Association of copeptin with recurrent stroke/MI in relation to other biomarkers. a HR and $95 \%$ CI. b HR and p values. $\mathrm{BDNF}=$ Brain-derived neurotrophic factor; $\mathrm{CRP}=\mathrm{C}$-reactive protein; DD = D-dimer; hFABP = heart-type fatty acid binding protein; NGAL = neutrophil gelatinase-associated lipocalin; NSE = neuron-specific enolase; nt-proBNP $=\mathrm{N}$-terminal pro-brain natriuretic peptide; $\mathrm{PC}=$ phosphorylcholine; $\mathrm{PZ}=$ protein $\mathrm{Z} ; \mathrm{TM}=$ thrombomodulin; TNF-R1 = TNF receptor 1 ; vWF $=$ von Willebrand factor. vascular deaths; 317 patients died of any cause. Copeptin levels at baseline were strongly correlated with 1 -year levels $(r=0.72, p<0.001)$, only moderately to age or baseline National Institutes of Health Stroke Scale scores $(r=0.26$ and 0.32 , respectively, both $\mathrm{p}<0.001$ ) but not to time from event to sampling.

After adjustment for age, sex, hypertension, diabetes, previous MI, previous stroke, previous peripheral vascular disease, smoking status, chronic heart failure, atrial fibrillation and therapy with antiplatelet agents, statins or antihypertensive agents, copeptin was predictive of re- current ischaemic stroke - hazard ratio (HR) per $\mathrm{SD}=$ $1.31,95 \%$ CI: $1.10-1.58, \mathrm{p}=0.004$; results were consistent for recurrent stroke/MI and even pronounced for vascular death (table 1). In addition, copeptin was also highly predictive of death of any cause ( $\mathrm{HR}=1.51,95 \%$ CI $1.34-$ $1.70, \mathrm{p}<0.0001)$. Analysis of levels by tertiles revealed similar results - stroke/MI: top tertile versus lowest tertile: $\mathrm{HR}=1.76,95 \%$ CI 1.17-2.66, $\mathrm{p}=0.007$; recurrent ischaemic stroke: $\mathrm{HR}=2.21,95 \% \mathrm{CI} 1.37-3.55, \mathrm{p}=0.002$, and vascular death: $\mathrm{HR}=2.77,95 \%$ CI $1.63-4.69, \mathrm{p}<$ 0.0001 . The predictive value of copeptin was stronger 
when we analysed associations of 1-year levels and recurrent vascular events (recurrent ischaemic stroke: $\mathrm{HR}=$ $1.70,95 \%$ CI 1.30-2.21, p < 0.0001; stroke or MI: HR = $1.60,95 \%$ CI 1.24-2.07, $\mathrm{p}<0.0001)$.

As compared to a panel of standard biomarkers related to inflammation (IL-6, C-reactive protein, neutrophil gelatinase associated lipocalin and TNF receptor 1), thrombosis or atherogenesis (fibrinogen, von Willebrand factor antigen, P-selectin, D-dimer, thrombomodulin and antiphosphorylcholine) and cardiac or neuronal function or injury (N-terminal pro-brain natriuretic peptide, hearttype fatty acid binding protein, brain-derived neurotrophic factor and neuron-specific enolase), copeptin was the strongest predictor of recurrent vascular events (fig. 1a, b).

\section{Conclusion}

In patients with TIA or minor ischaemic stroke, copeptin predicted recurrent vascular events and all-cause death. Further validation and mechanistic studies are required.

\section{4}

Age-Specific Risk and Severity of Bleeding on Aspirin-Based Secondary Prevention in Transient Ischaemic Attack and Ischaemic Stroke: A Population-Based Study

L. Li, O.C. Geraghty, Z. Mehta, P.M. Rothwell,

Nuffield Department of Clinical Neurosciences, John Radcliffe Hospital, University of Oxford, Oxford, UK

\section{Background}

Use of aspirin in the secondary prevention after transient ischaemic attack (TIA) and ischaemic stroke is based on randomised controlled trials (RCTs) done almost entirely in patients aged $<75$ years. However, patients aged $\geq 75$ years now constitute almost half of all those with TIA or ischaemic stroke. Reliable data on the risk and severity of bleeds in this older age group are therefore important.

\section{Methods}

We prospectively determined the age-specific rates and severity of all bleeding events necessitating medical attention during follow-up in all patients on aspirinbased antiplatelet treatment after TIA or ischaemic stroke from the first 10 years of a population-based study (Oxford Vascular Study, OXVASC). Face-to-face interview, all-hospital and primary care diagnostic coding and blood transfusion data in Oxfordshire were used to identify all bleeding events requiring medical attention. The severity of all bleeding events was classified by the CURE (Clopidogrel in Unstable Angina to Prevent Recurrent Events) trial criteria. Major bleeding was defined as substantially disabling with persistent sequelae, intraocular bleeding leading to significant loss of vision or bleeding requiring transfusion of 2 or more units of blood. It was further classified as fatal or life-threatening if it led to a decrease in haemoglobin of $5 \mathrm{~g} / \mathrm{dl}$, if it caused significant hypotension requiring intravenous inotropes or surgical intervention, if it resulted in symptomatic intracranial haemorrhage, or if it necessitated transfusion of 4 or more units of blood. Bleeding events that required medical attention but did not fulfil the criteria for major bleeding were defined as minor bleeding. Bleeds secondary to major trauma, major surgical procedures or coagulation abnormalities were not included. Bleeding sites were defined as intracranial (intracerebral, subdural or subarachnoid haemorrhage) and extracranial (upper and lower gastrointestinal, genitourinary and other). We compared rates to pooled estimates of major bleeding rates in all RCTs of aspirin for the secondary prevention after TIA and ischaemic stroke.

\section{Results}

Of 2,072 patients on antiplatelet treatment (1962/ 2,072, $94.7 \%$ aspirin based), 984 patients (47.5\%) were $<75$ years and $1,088(52.5 \%) \geq 75$ years. Compared to RCTs (mean age: 63 years), patients in OXVASC were much older. During 8,799 patient-years of follow-up, 254 patients with first bleeding events (117 major bleedings) presented to medical attention, of whom 193 (76\%) were hospitalised acutely. The absolute risks of major bleeding increased sharply with age (fig. 1 ). At age $<75$ years (mean $=62$ ), the annual rate of major bleeding during a 3-year follow-up was $1.1 \%$ (27 events; 95\% CI 0.7-1.6\%), which was highly comparable to the pooled rate from 14 aspirin trials (mean age: 63 years; mean follow-up: 2.5 years; 520 
Table 1. Ten-year bleeding risks stratified by age and bleeding severity

\begin{tabular}{lcclc}
\hline & $\begin{array}{l}<5 \text { years: events, } \mathrm{n} \\
(4,571 \text { patient-years })\end{array}$ & $\begin{array}{l}\geq 75 \text { years: events, } \mathrm{n} \\
(3,273 \text { patient-years })\end{array}$ & $\begin{array}{l}\text { Hazard ratio } \\
(95 \% \mathrm{CI})\end{array}$ & $\begin{array}{l}\mathrm{p} \\
\text { value }\end{array}$ \\
\hline All bleeds & 104 & 150 & $1.77(1.37-2.27)$ & $<0.0001$ \\
Minor & 71 & 66 & $1.14(0.81-1.60)$ & 0.44 \\
Major non-fatal & 28 & 58 & $2.46(1.57-3.88)$ & $<0.0001$ \\
Fatal & 5 & 26 & $6.50(2.49-16.97)$ & $<0.0001$ \\
\hline
\end{tabular}

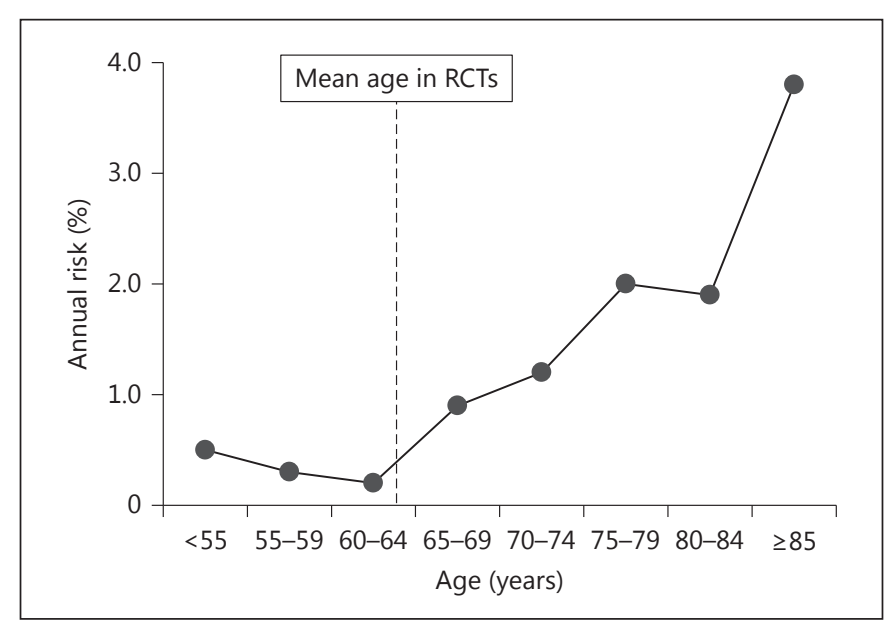

Fig. 1. Annual risks of major bleeding stratified by age in OXVASC.

major bleeds/52,984 patient-years, $1.0 \%, 95 \%$ CI 0.8-1.1). However, the risk of major bleeding was significantly higher $(\mathrm{p}<0.0001)$ in study patients aged $\geq 75$ years $(68$ events; $3.2 \%$, 95\% CI 2.5-4.1) and the bleeds were more severe (table $1 ; \mathrm{p}<0.0001$ ).

\section{Conclusions}

The absolute risk of major bleeding on aspirin-based antiplatelet treatment in patients with TIA and ischaemic stroke aged $<75$ years is consistent with the risk reported in RCTs. However, risks are much higher and more severe in older patients, for whom there are no data from RCTs on the overall balance of risk and benefit.

\section{5 \\ Long-Term Outcome in Asymptomatic Intracranial Atherosclerosis (The Barcelona-AsIA Study)}

E. López-Cancio ${ }^{a}$, L. Dorado ${ }^{a}$, G. Pera ${ }^{b}$, P. Torán ${ }^{b}$, M. Alzamora ${ }^{b}$, M. Hernández-Pérez ${ }^{a}$, M. Gomis ${ }^{a}$, N. Pérez de la Ossa ${ }^{a}$, J.F. Arenillas ${ }^{c}$, A. Dávalos ${ }^{a}$

${ }^{a}$ Hospital Universitari Germans Trias i Pujol, and bUnitat de Suport a la Recerca Metropolitana Nord, IDIAP Jordi Gol, Barcelona, and ${ }^{\mathrm{C}} \mathrm{Hospital}$ Clinico Valladolid, Valladolid, Spain

\section{Background/Aim}

Symptomatic intracranial atherosclerosis is one of the most important causes of stroke worldwide and it is associated with a high risk of stroke recurrence even with aggressive medical treatment. Knowledge on symptomatic intracranial atherosclerosis is increasing in the last decades. However, data of population studies on the prognosis of asymptomatic intracranial atherosclerosis are lacking. We aimed to study the prognostic impact of intracranial atherosclerosis from its asymptomatic stage in a population-based cohort.

\section{Methods}

The Barcelona-AsIA (Asymptomatic Intracranial Atherosclerosis) study is a prospective population-based study aimed to determine the prevalence of asymptomatic intracranial atherosclerosis, its associated clinical, molecular and genetic risk factors, and its prognostic impact. The AsIA study included a random sample of 933 Caucasian subjects $>50$ years with a moderate-high vascular risk (assessed by REGICOR score, which is the Framingham risk score validated for the Spanish population) and without a history of stroke or coronary disease. Subjects were consecutively recruited from March 2007 to June 2010. At the baseline visit, extracranial carotid artery stenosis (ECAS) and intracranial atherosclerosis 
Fig. 1. Survival curves for both primary and secondary outcomes in the different groups of atherosclerosis location. log-rank (Mantel-Cox): $\mathrm{p}<0.001$. a Combined outcome: stroke, acute coronary syndrome and/or vascular death. b Stroke.

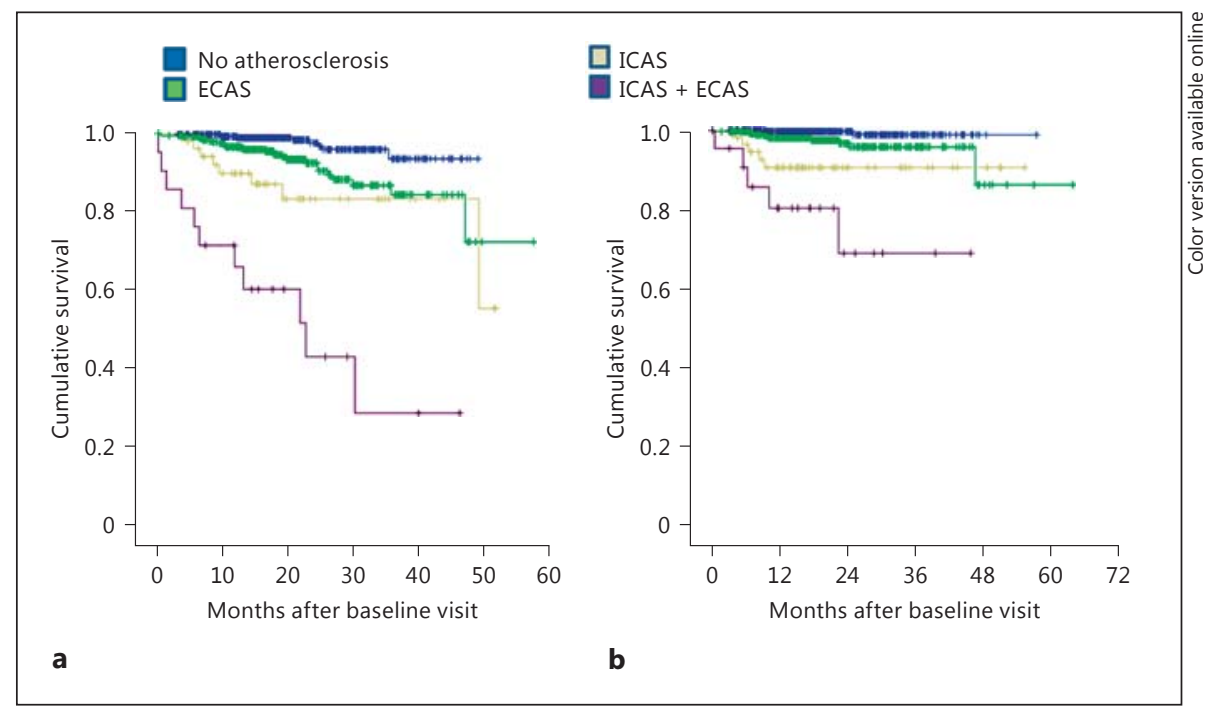

(ICAS) were screened by color-coded duplex ultrasound, being moderate-severe stenosis confirmed by MR angiography. Subjects are being contacted every 6 months and hospital records checked to assess the incidence of vascular events and/or death. Events are adjudicated by a committee blinded to clinical/atherosclerosis data. The follow-up period is projected for 5 years from the baseline visit, so here we present preliminary results of the followup study. Primary outcome was defined as the occurrence of any vascular event (stroke, acute coronary syndrome and/or vascular death) and secondary outcome as the occurrence of stroke. We assessed Kaplan-Meier curves (log-rank test) and constructed Cox regression models adjusted by age, sex, REGICOR score, presence of carotid plaques and presence of intracranial stenosis. REGICOR scores are calculated based on age, sex, diabetes, smoking, blood pressure and cholesterol levels.

\section{Results}

The mean age of the study participants was 66.4 years and $64 \%$ were male. At the baseline visit, 80 of the 933 subjects were found to have asymptomatic intracranial stenosis (ICAS group), 427 had at least one extracranial carotid plaque (ECAS group) and 449 had neither carotid plaques nor intracranial stenosis (no atherosclerosis group); 23 subjects had both extracranial and intracranial lesions (ECAS + ICAS group). The mean follow-up period of the entire cohort at the moment of the present analysis was $19.8( \pm 10.5)$ months. During this time, 23 subjects had a stroke, 28 had an acute coronary syndrome and 26 died (6 were deaths from vascular origin). Survival curves for both primary and secondary outcomes with respect to atherosclerosis location are presented in figure 1. After multivariate Cox regression analyses, independent predictors for overall vascular events were age (hazard ratio, HR 1.06, 95\% CI 1.03-1.11), presence of intracranial stenosis (HR 3.35, 95\% CI 1.84-6.08) and presence of carotid plaques (HR 2.65, 95\% CI 1.29-5.46). Independent predictors for stroke were also age (HR 1.08, 95\% CI 1.02-1.14), presence of intracranial stenosis (HR $4.59,95 \%$ CI 1.92-10.95) and presence of carotid plaques (HR 3.85, 95\% CI 1.09-13.5). The REGICOR score did not remain as an independent predictor in adjusted models.

\section{Conclusions}

In this population-based cohort study, we found that the presence of asymptomatic intracranial stenosis was associated with a higher risk of future vascular events, especially of stroke. This was independent of conventional vascular risk factors (REGICOR) and the presence of extracranial carotid plaques. These findings might have important implications for the development of primary prevention strategies. 


\section{6}

\section{Telemetric Bluetooth Home Blood Pressure Monitoring in Patients with Transient Ischaemic Attack and Minor Stroke: Feasibility and Blood Pressure Control}

N.G. Lovett, M. Wilson, L.E. Silver, F.C. Cuthbertson, J. Glennon, A.J.S. Webb, U. Fischer, L. Tarassenko, P.M. Rothwell, on behalf of the Oxford Vascular Study

University of Oxford, Oxford, UK

\section{Background}

Hypertension is a major modifiable risk factor for recurrent stroke and vascular disease. However, the prevalence of hypertension is often underestimated, due in part to reliance on single readings in the clinic, with rates of 'masked' hypertension [patients who have normal clinic blood pressure (BP) readings but elevated home readings] ranging from 10 to $30 \%$ in different populations. Moreover, although randomised trials show that blood pressure lowering is highly effective in the secondary prevention of stroke, rates of control in practice are low. Home BP monitoring is useful as it reduces the effect of white coat hypertension, allows multiple readings to be collected, giving better prognostic accuracy than office BP measurements alone, and helps quantify BP variability. Patients are also able to see the direct effect of antihypertensive medication on their BP, which might help to aid compliance. We, therefore, sought to determine the feasibility of using Bluetooth telemetric home monitoring of BP to improve control after a transient ischaemic attack (TIA) or minor stroke.

\section{Methods}

We studied consecutive eligible patients with a recent TIA or non-disabling stroke in a population-based study. After prescription of initial BP-lowering therapy, if required, patients measured their BP 3 times over 10 min on 3 occasions each day at home with a Bluetooth-equipped monitor ( $\mathrm{t}+$ Medical, Abingdon, UK) for 1-3 months, depending on control. Measurements transmitted automatically in real time using mobile phone technology were checked daily on a secure web page. If $\mathrm{BP}$ was consistently above 130/80 mm Hg or below 100/60 mm Hg, antihypertensive therapy was adjusted. Patients underwent 24-hour ambulatory $\mathrm{BP}$ monitoring at 1 month, and $\mathrm{BP}$ control was assessed again in the clinic at 3 months. Patients' views were evaluated by an anonymised questionnaire at 1 month.

\section{Results}

Of 897 eligible patients, 845 agreed to participate (94\%; mean $/$ SD age $=69 / 13 ;$ range $=21-98$ years; $23 \% \geq 80$ years), of whom 12 (1.4\%) withdrew within 1 month. Masked hypertension (BP $\leq 140 / 90$ at initial assessment and mean $\mathrm{BP}>135 / 85$ across the first 3 days of monitoring; AHA definition) was found in 266 (32\%) patients. Monitoring resulted in $77 \%$ of patients having changes in $\mathrm{BP}$ medication. Of patients in whom changes were made, $72 \%$ initiated/increased dose and $24 \%$ stopped/decreased dose between 0 and 1 month and 66\% initiated/increased dose and 31\% stopped/decreased dose between 1 and 3 months. Mean (SD) BP was 150 (21) mm Hg (systolic) and $82(11.3) \mathrm{mm} \mathrm{Hg}$ (diastolic) at study entry and 126 (15.8) $\mathrm{mm} \mathrm{Hg}$ (systolic) and 72 (10.0) $\mathrm{mm} \mathrm{Hg}$ (diastolic) at the 3-month follow-up.

A mean of 8.7 readings per patient per day was achieved. Among the 460 (55\%) patients who responded to the questionnaire, 415 (90\%) approved of intensive monitoring and $407(88 \%)$ were reassured by the remote surveillance; 78 (17\%) felt the process made them feel anxious and 69 (15\%) felt it was too time-consuming.

At baseline, $46 \%$ of patients were not taking any antihypertensive medication, $19.7 \%$ were taking $1,19.2 \%$ were taking 2 and $15.2 \%$ were taking 3 or more antihypertensive drugs. At 1 month, $21.3 \%$ were not taking any, $15.3 \%$ were taking $1,32.7 \%$ were taking 2 and $30.7 \%$ were taking 3 or more antihypertensive drugs. At their 12 -month follow-up, $31.3 \%$ of patients were not taking any, $13.6 \%$ were taking 1,25.9\% were taking 2 and $29.8 \%$ were taking 3 or more antihypertensive medications.

\section{Conclusion}

Telemetric home BP monitoring is feasible and well tolerated in patients with TIA and minor stroke irrespective of age. Monitoring detects high levels of masked hypertension, provides information regarding titration of medication in the majority of patients and is associated with good BP control at 3 months. Home monitoring serves to raise awareness and educate patients, as they are able to see the direct effect that antihypertensive medication has on their BP. This aids long-term compliance, which is reflected by the majority of patients remaining on 2 or 3 antihypertensive agents at 6 months and 1 year. 
Fig. 1. Associations of hyperacute and acute SD-SBP (quintiles) and primary outcome [courtesy of Lancet Neurol 2014;13: 364-373] with variability on day 1 (a-c) and days 2-7 (d-f). a, d Model 1. b, e Model 2. c, f Model 3.

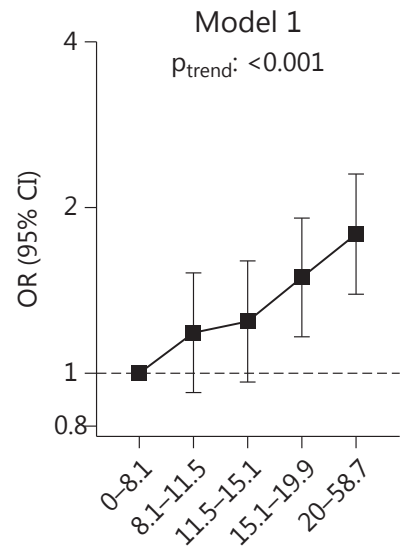

a

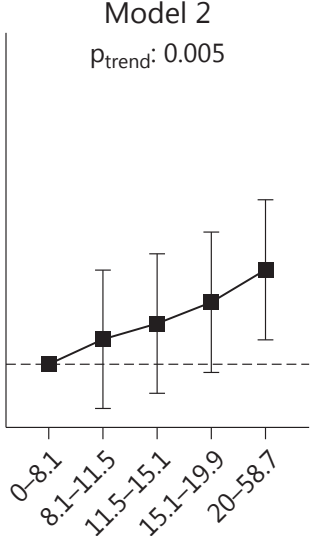

Fifths of SD-SBP

b

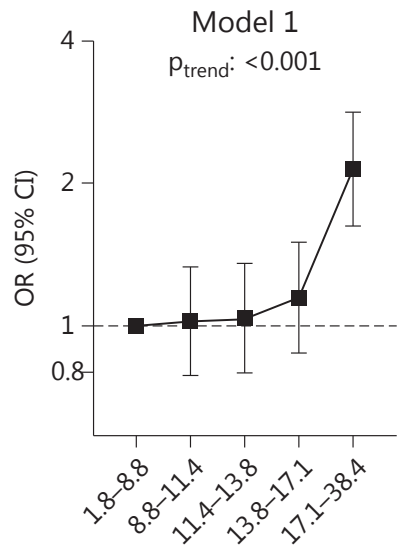

d

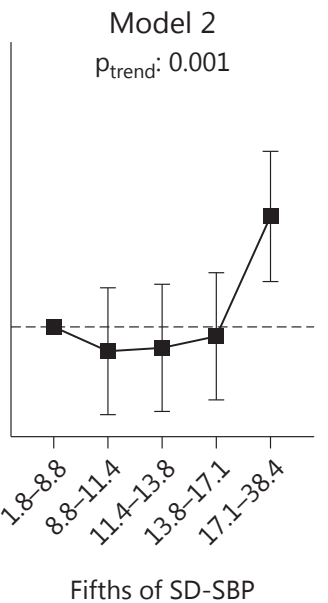

e
Model 3

$p_{\text {trend }}: 0.017$

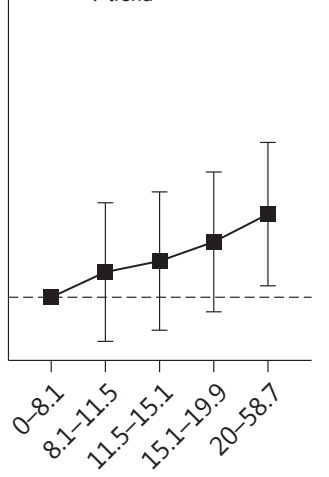

c

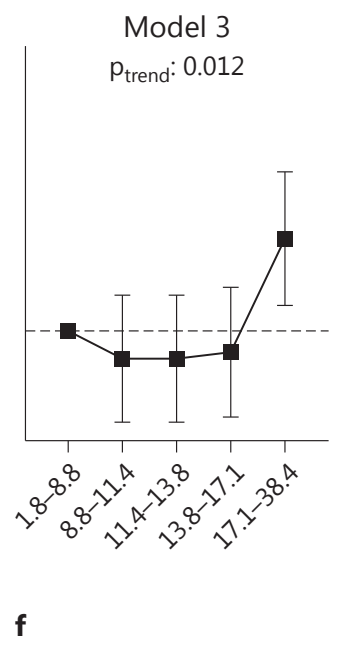

7

\section{Usefulness of Various Parameters of Systolic Blood Pressure Variability in Predicting Outcome in Acute Intercerebral Haemorrhage: Analysis of the INTERACT2 Study}

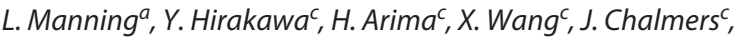
T.G. Robinson ${ }^{a}$, P. Rothwell ${ }^{b}$, C.S. Anderson', on behalf of the INTERACT2 Investigators

aDepartment of Cardiovascular Sciences and NIHR Biomedical Research Unit in Cardiovascular Disease, University of Leicester, Leicester, and bStroke Prevention Research Unit, University Department of Clinical Neurology, John Radcliffe Hospital, Oxford, UK; 'The George Institute for Global Health, University of Sydney and Royal Prince Alfred Hospital, Sydney, N.S.W., Australia

\section{Background}

High blood pressure (BP) predicts a poor outcome in intracerebral haemorrhage (ICH), but the influence of $\mathrm{BP}$ variability (BPV) has been less certain. We examined the significance of BPV in the INTERACT2 study.

\section{Methods}

INTERACT2 was an international, multicentre, randomised, controlled trial of early $(<6 \mathrm{~h})$ intensive [systolic BP (SBP) target $<140 \mathrm{~mm} \mathrm{Hg}$ within $1 \mathrm{~h}$ ] versus guideline-recommended (SBP target $<180 \mathrm{~mm} \mathrm{Hg}$ ) BP lowering in 2,839 hypertensive (SBP $150-220 \mathrm{~mm} \mathrm{Hg}$ ) 
Table 1. Associations of 1-SD increment in systolic BPV on the primary outcome [Courtesy of Lancet Neurol 2014;13:364-373]

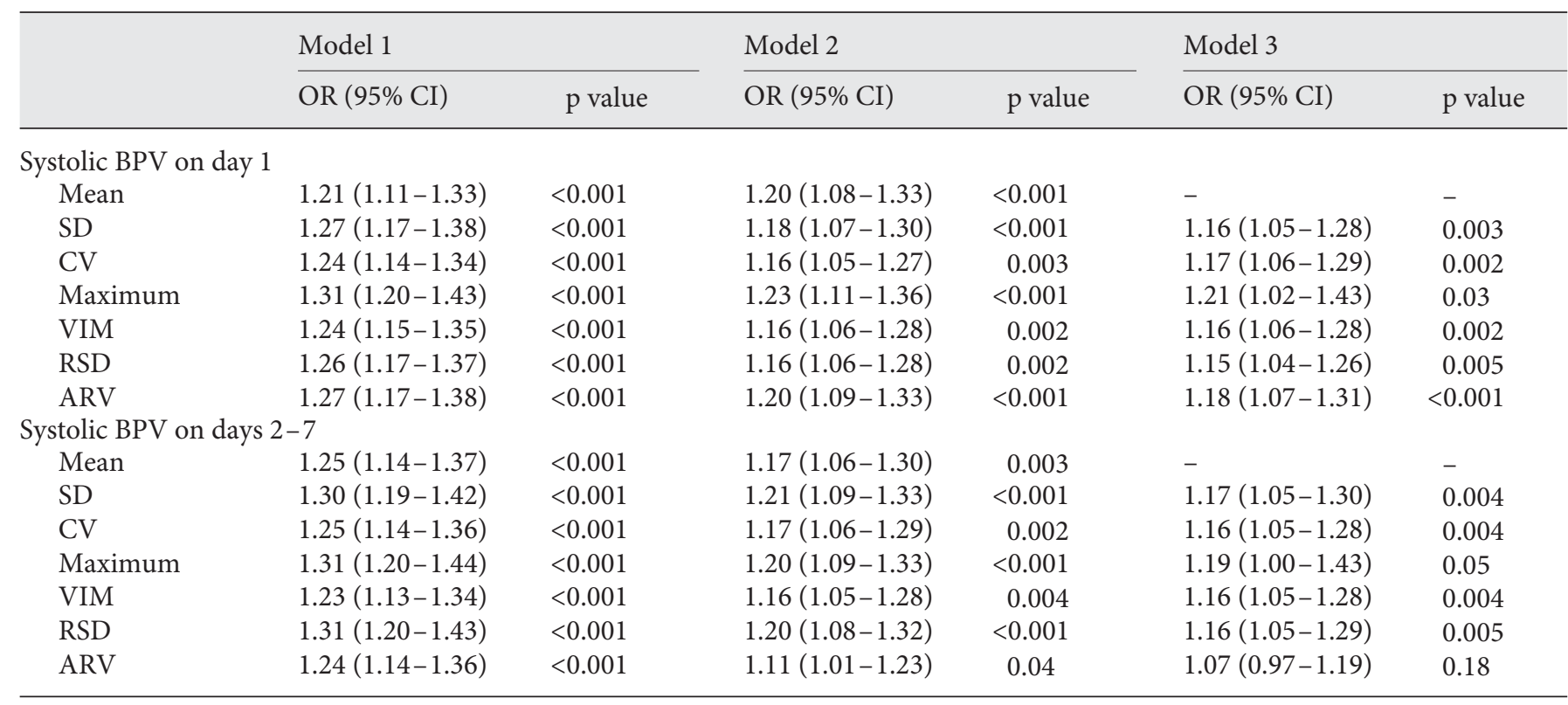

$\mathrm{ARV}=$ Average real variability; $\mathrm{CV}=$ coefficient of variation; $\mathrm{RSD}=$ residual $\mathrm{SD} ; \mathrm{VIM}=$ variation independent of mean.

patients with mainly mild-moderate severity of acute spontaneous ICH. BPV was based on standardised readings: 5 on day 1 (hyperacute phase) and 12 on days 2-7 (acute phase). Associations of BPV with poor outcome (death or dependency; modified Rankin scale score $\geq 3$ ) at 90 days were estimated in staged logistic regression models: model 1 adjusted for age, sex and treatment group; model 2, adjusted for all covariables in model 1, plus region, National Institutes of Health Stroke Scale score and haematoma volume, and model 3 , adjusted for all covariables in model 2 plus mean BP. The key index of BPV was standard deviation (SD) for SBP (SD-SBP), which was categorised into quintiles with odds ratios (OR) and 95\% confidence intervals (CI) estimated using the lowest 5 th as reference.

\section{Results}

We studied 2,645 (93.2\%) and 2,347 (82.7\%) INTERACT2 participants in the hyperacute and acute phases of $\mathrm{ICH}$, respectively. SD-SBP showed a significant linear association with outcome in the hyperacute (highest 5 th adjusted OR 1.41, 95\% CI 1.05-1.9; $\left.\mathrm{p}_{\text {trend }}=0.02\right)$ and acute (OR 1.57, 95\% CI 1.14-2.17; $\mathrm{p}_{\text {trend }}=0.012$ ) phases (fig. 1). In the hyperacute phase, all parameters of systolic BPV were significantly associated with outcome, and in the acute phase, all except average real variability remained significantly associated (table 1). The strongest BPV parameters that predicted outcome were maximum SBP (hyperacute phase) and SD-SBP (acute phase). Diastolic $\mathrm{BPV}$ and mean arterial pressure were weakly associated with outcome.

\section{Conclusion}

Peaks in SBP, defined by systolic BPV, are an independent predictor of outcome in ICH. Mean and maximal SBP were defined as the most meaningful BPV parameters for clinical practice; whereas diastolic BP and mean arterial pressure had limited prognostic significance. The potential benefits of early BP-lowering treatment to a target $(140 \mathrm{~mm} \mathrm{Hg})$ SBP may be enhanced by smooth and sustained control, with avoidance of peaks in SBP in the setting of acute ICH. 


\section{Thirty-Year Trends in First-Time Hospitalization for Subarachnoid Hemorrhage and Subsequent Short- and Long-Term Mortality: A Danish Nationwide Study}

T.P. Ottosen ${ }^{a, c}$, M. Schmidt ${ }^{a}$, J.B. Jacobsen ${ }^{a}$, H.H. Stabel ${ }^{d}$ J.F. Nielsen ${ }^{d}$, H.E. Bøtker ${ }^{b}$, S.P. Johnsen ${ }^{a}$, H.T. Sørensen ${ }^{a}$

Departments of a Clinical Epidemiology, ${ }^{b} \mathrm{C}$ ardiology and 'Neurology, Aarhus University Hospital, and ${ }^{\mathrm{d}} \mathrm{Hammel}$ Neurorehabilitation Center and University Research Clinic, Aarhus University, Aarhus, Denmark

\section{Background}

Subarachnoid hemorrhage (SAH) accounts for about $5 \%$ of all stroke episodes. Nearly one third of all strokerelated years of potential life lost before age 65 years is attributable to $\mathrm{SAH}$, which makes the impact on the loss of productive life years similar to that of ischemic stroke [1]. Data on long-term trends in incidence and case fatality of SAH are sparse. Also, the prognostic impact of comorbidity has been examined for ischemic stroke and intracerebral hemorrhage, but not yet for SAH. We, therefore, conducted a population-based cohort study to examine nationwide trends in first-time hospitalization and case fatality following SAH in Denmark between 1983 and 2012.

\section{Methods}

Denmark has approximately 5.6 million inhabitants and the Danish National Health Service provides universal tax-supported health care, guaranteeing unfettered access to general practitioners and hospitals. In this nationwide study, we used the Danish National Registry of Patients to identify all patients with a first-time hospitalization for SAH during 1983-2012. The database is based on discharge diagnoses and covers all Danish hospitals. We used the Danish Civil Registration System to obtain the vital status of the patients in the years following their hospitalization. This linkage is possible by use of the central personal registration number system, which assigns a unique personal identification number to each Danish citizen at birth and to residents upon immigration. Ageadjusted standardized hospitalization rates for SAH were calculated using the year 2000 population as reference. We computed age-standardized 30-day, 1-year and 5-year mortality rates by sex. Calendar periods of diagnosis
(1983-1987, 1988-1992, 1993-1997, 1998-2002, 20032007 and 2008-2012) were compared using mortality rate ratios based on Cox regression. We used the Charlson Comorbidity Index score to describe comorbidity, separating the scores into four categories (no, moderate-, severe- and very severe comorbidity). The index score predicts 1-year mortality among patients who may have a range of comorbid conditions. It includes 19 conditions, including diabetes, myocardial infarction and chronic lung disease. It is constructed based on the hospitalization history, which was conducted for each patient going back to 1977.

\section{Results}

We identified 18,120 first-time hospitalizations for SAH. Women accounted for $58.5 \%$ of all cases. From 1983 to 2012, the median age increased from 54 to 64 years for women and from 53 to 62 years for men.

Within the 30-year period, the proportion of patients having no comorbidity before admission decreased from 86.3 to $67.1 \%$. Patients who had moderate comorbidity increased $6.9 \%$ (from 7.2 to $14.1 \%$ ), whereas those with very severe comorbidity also increased $6.9 \%$ (from 1.7 to $8.6 \%)$.

The annual standardized rate of first-time hospitalization for SAH per 100,000 person-years increased during this 30 -year period overall by $25 \%$ from 12 (95\% CI $11-$ 13) to 15 (95\% CI 14-16). The hospitalization rate peaked in year 2007 and was followed by a minor decrease towards the end of the period. The hospitalization rate was higher among women than men within the entire period, but the developments over time were comparable (fig. 1).

Thirty-day, 1-year and 5-year mortality declined from $38.0,43.8$ and $51.2 \%$ in $1983-1987$ to $25.2,31.7$ and $37.8 \%$ in 2008-2012, respectively (fig. 2). With the 1983-1987 calendar period as reference, the corresponding mortality rate ratios for patients admitted in 2008-2012, adjusted for age, sex and Charlson Comorbidity Index, were 0.52 (95\% CI 0.48-0.58), 0.55 (95\% CI 0.44-0.69) and 0.51 (95\% CI 0.33-0.54).

In 2003-2007, SAH patients without comorbidity had an overall $24.6 \% 30$-day mortality risk, increasing to $28.4 \%$ at 1 year and $34.2 \%$ at 5 years. The corresponding mortality risks associated with very severe comorbidity were $40.8,54.9$ and $75.6 \%$, respectively. Overall, higher severity of comorbidity was associated with increased risk of both short- and long-term mortality. 


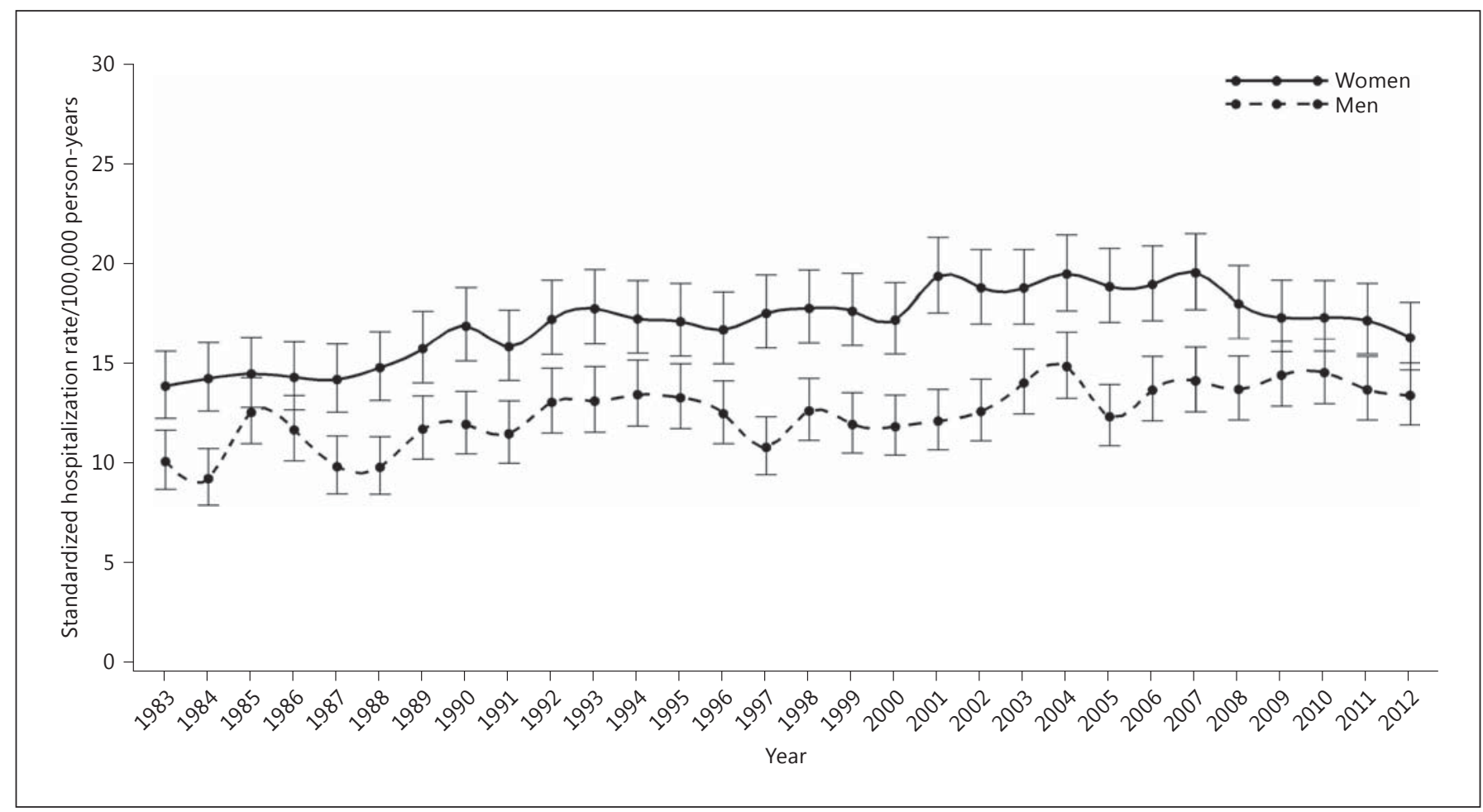

Fig. 1. Observed age-standardized hospitalization rates (per 100,000) for SAH in men and women.

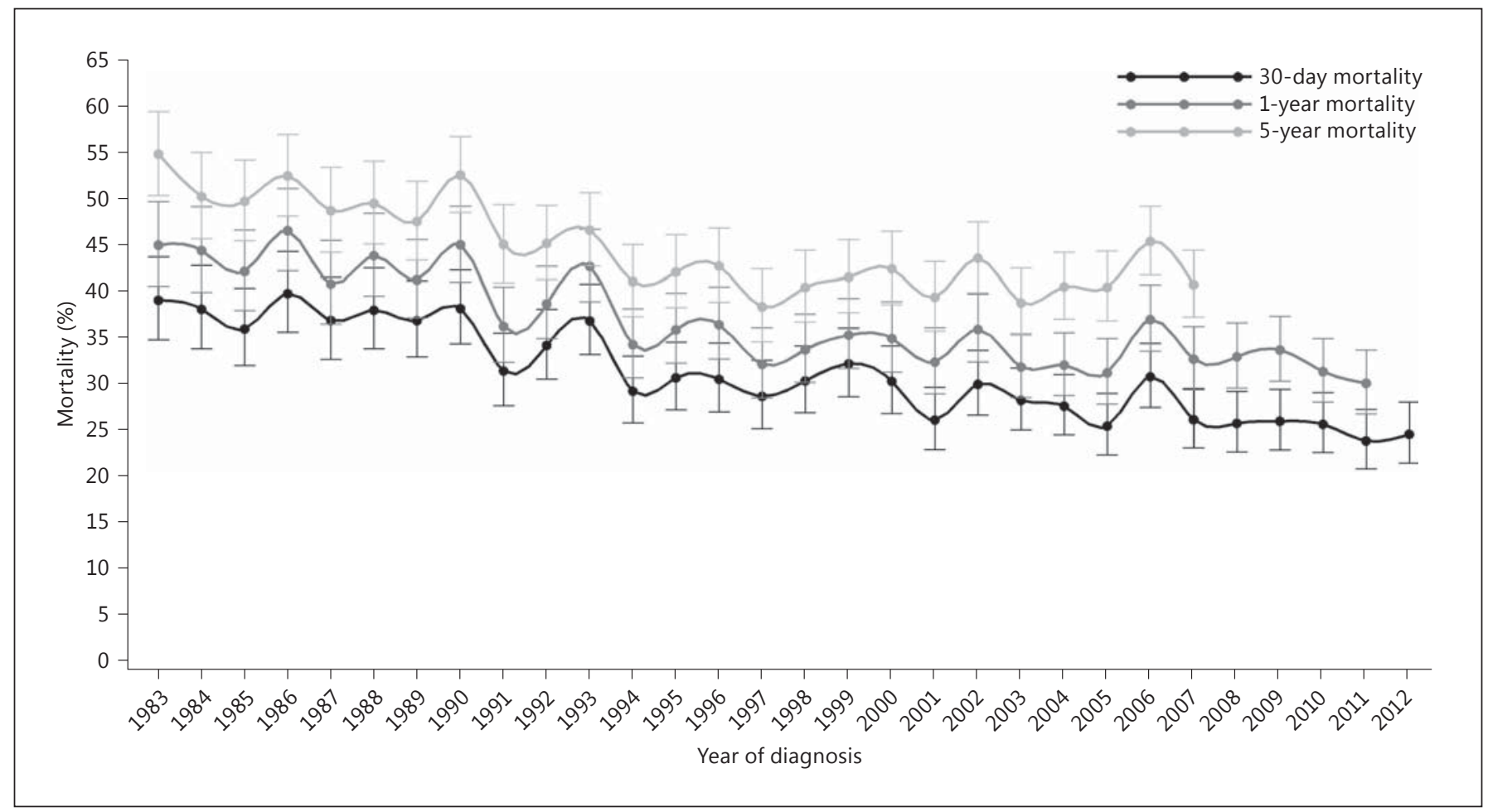

Fig. 2. Overall age-standardized 30-day, 1-year and 5-year mortality rates, according to the year of diagnosis. 
In the period 1983-1987, the adjusted 1-year mortality risk ratio among those with moderate comorbidity was 1.17 (95\% CI 0.90-1.52) compared with 1.18 (95\% CI $0.98-1.43$ ) in the period 2008-2012. Overall, the impact of comorbidity on mortality did not change within the observed period.

\section{Conclusions}

The median age at first-onset SAH among both women and men increased by approximately 10 years in Denmark during the period 1983-2012. There was an increase in the rate of first-time hospitalization for SAH overall by $25 \%$. Further, the proportion of patients with very severe comorbidity increased by more than a factor of 5 and constituted approximately 1 out every 10 patients at the end of the study period. Despite this increase, there were no major changes in the impact of comorbidity on mortality over time. Driven by a reduction in 30-day mortality, both short- and long-term survival after SAH improved markedly during the 30 -year period.

\section{Reference}

1 Shea AM, Reed SD, Curtis LH, Alexander MJ, Vilani JJ, Schulman KA: Characteristics of nontraumatic subarachnoid hemorrhage in the United States in 2003. Neurosurgery 2007;61:1131-1137.

\section{9}

\section{The Montreal Cognitive Assessment Test Is More Strongly Associated with White Matter Ultrastructural Damage than the Mini-Mental State Examination in Patients with Vascular Mild Cognitive Impairment: The Vascular Mild Cognitive Impairment in Tuscany Study}

M. Pasi ', E. Salvadori' , A. Poggesia , G. Pracucci ${ }^{a}$, L. Ciollia, A. Del Bene ${ }^{a}$, S. Marini ${ }^{a}$, S. Nannucci ${ }^{a}$, F. Pescini ${ }^{a}$, R. Valenti ${ }^{a}$, S. Diciotti ${ }^{d}$, M. Mascalchib ${ }^{b}$ D. Inzitari ${ }^{a}$, L. Pantonic, on behalf of the VMCI Study Group

aNeuroscience Section, NEUROFARBA Department, University of Florence, ${ }^{b}$ Quantitative and Functional Neuroradiology Research Program, Meyer Children Hospital and Careggi General Hospital, and 'Stroke Unit and Neurology, Azienda Ospedaliero Universitaria Careggi, Florence, and 'Department of Electrical, Electronic and Information Engineering 'Guglielmo Marconi', Alma Mater Studiorum-University of Bologna, Cesena, Italy

\section{Background}

The Montreal Cognitive Assessment (MoCA) test is a simple screening tool with good sensitivity and specificity in detecting mild cognitive impairment (MCI) [1]. Moreover, the MoCA test has been proposed as a valid instrument to evaluate patients with vascular cognitive impairment because it includes items reflecting mental speed and executive functions. The widely used MiniMental State Examination (MMSE) has been reported to be poor in detecting MCI and suboptimal in assessing cognitive abnormalities associated with cerebrovascular disease $[1,2]$.

Diffusion tensor imaging (DTI) is an MRI technique that allows to study in vivo the tissue microstructure and is often applied to study cerebral white matter (WM). It has been shown that microstructural changes depicted by diffusion images predict faster decline in psychomotor speed, executive functions and working memory regardless of conventional MRI findings [3].

We aimed at assessing whether WM ultrastructural damage in patients with MCI and small vessel disease (SVD) is more strongly reflected by MoCA than MMSE performances taking into account other neuroimaging variables.

\section{Methods}

The Vascular Mild Cognitive Impairment (VMCI) in Tuscany study is a multicenter, prospective, observational study designed to estimate the effect of clinical, neuroimaging and biological markers of SVD in predicting the transition from VMCI to dementia. To be included, patients had to be classified as affected by VMCI with SVD according to the following criteria: (1) MCI defined according to Winblad criteria and (2) MRI evidence of moderate-to-severe WM hyperintensities according to the modified version of the Fazekas scale. The VMCITuscany methodology and study protocol have been extensively described in a previous paper [4].

For all patients, demographic variables (age, education and gender) were collected. Regarding neuroimaging evaluation, lacunar infarcts, global cortical atrophy, medial temporal lobe atrophy (MTLA), and DTI parameters (mean diffusivity, MD, and fractional anisotropy, FA) were assessed. All patients were evaluated with MoCA (0-30) and MMSE (0-30).

Bivariate statistical analyses (independent $t$ test, ANOVA and Pearson's r) were used to evaluate associations of 
Table 1. Univariate analysis: association between demographic and neuroimaging variables and MMSE and MoCA

\begin{tabular}{|c|c|c|c|c|}
\hline Variables & MMSE & $\mathrm{p}$ & MoCA & $\mathrm{p}$ \\
\hline Age & $-0.213^{\mathrm{a}}$ & 0.037 & $-0.409^{a}$ & $<0.001$ \\
\hline Education (years) & $0.389^{a}$ & $<0.001$ & $0.471^{\mathrm{a}}$ & $<0.001$ \\
\hline \multicolumn{5}{|l|}{ Gender } \\
\hline Female & $25.4 \pm 3.4$ & $0.017^{b}$ & $17.8 \pm 5.6$ & $0.042^{\mathrm{b}}$ \\
\hline Male & $26.9 \pm 2.8$ & & $20.1 \pm 5.6$ & \\
\hline \multicolumn{5}{|l|}{ Lacunar infarcts } \\
\hline 0 & $25.5 \pm 4.0$ & $\mathrm{NS}^{\mathrm{c}}$ & $19.7 \pm 5.3$ & $\mathrm{NS}^{\mathrm{c}}$ \\
\hline $1-3$ & $26.3 \pm 3.2$ & & $18.3 \pm 5.5$ & \\
\hline$>3$ & $26.6 \pm 2.4$ & & $19.7 \pm 5.5$ & \\
\hline \multicolumn{5}{|l|}{ Fazekas } \\
\hline Grade 2 & $26.6 \pm 3.1$ & $\mathrm{NS}^{\mathrm{b}}$ & $20.2 \pm 5.2$ & $\mathrm{NS}^{\mathrm{b}}$ \\
\hline Grade 3 & $26.0 \pm 3.3$ & & $18.3 \pm 5.5$ & \\
\hline \multicolumn{5}{|l|}{ Global atrophy } \\
\hline Grade 1 & $28.4 \pm 1.2$ & $0.001^{\mathrm{c}}$ & $23.3 \pm 3.0$ & $0.015^{\mathrm{c}}$ \\
\hline Grade 2 & $26.1 \pm 3.0$ & & $18.5 \pm 5.3$ & \\
\hline Grade 3 & $24.1 \pm 4.5$ & & $17.6 \pm 6.4$ & \\
\hline MTLA & $-0.293^{a}$ & 0.004 & $-0.307^{a}$ & 0.003 \\
\hline $\mathrm{MD}$ & $-0.242^{\mathrm{a}}$ & 0.017 & $-0.371^{\mathrm{a}}$ & $<0.001$ \\
\hline FA & $0.215^{\mathrm{a}}$ & 0.036 & $0.222^{\mathrm{a}}$ & 0.032 \\
\hline
\end{tabular}

${ }^{a}$ Pearson's r. ${ }^{b}$ Student's t test. ${ }^{c}$ ANOVA. Statistically significant results are in bold.

Table 2. Partial correlation analysis: association between DTI parameters and MoCA/MMSE independently from demographic and neuroimaging variables

\begin{tabular}{|c|c|c|c|c|c|c|c|c|}
\hline & \multicolumn{4}{|c|}{ Model 1} & \multicolumn{4}{|c|}{ Model 2} \\
\hline & \multicolumn{2}{|l|}{$\mathrm{MD}$} & \multicolumn{2}{|l|}{ FA } & \multicolumn{2}{|l|}{$\mathrm{MD}$} & \multicolumn{2}{|l|}{ FA } \\
\hline & $\mathrm{r}$ & $\mathrm{p}$ & $r$ & $\mathrm{p}$ & $\mathrm{r}$ & $\mathrm{p}$ & $\mathrm{r}$ & $\mathrm{p}$ \\
\hline MMSE & -0.203 & 0.054 & 0.218 & 0.038 & -0.172 & 0.112 & 0.270 & 0.011 \\
\hline $\mathrm{MoCA}$ & -0.320 & 0.002 & 0.246 & 0.019 & -0.274 & 0.010 & 0.244 & 0.023 \\
\hline
\end{tabular}

Model 1: adjusted for age, education and gender. Model 2: model 1 plus adjustment for lacunar infarcts, WM hyperintensities, global atrophy, MTLA. Statistically significant results are in bold.

demographic and neuroimaging variables with MoCA and MMSE.

We performed four linear regression models to evaluate associations of MoCA and MMSE with each neuroimaging variable, which resulted in significant differences in univariate analysis independently from age, education and gender. In order to evaluate which test was more strongly associated with each DTI parameter, we also per- formed 2 models of partial correlation analysis. In the first model, we adjusted only for demographic variables. In the second model, we adjusted for demographics and all neuroimaging variables.

\section{Results}

Ninety-six patients (mean age \pm SD: $75.2 \pm 7.1$, females $43.7 \%$, mean years of education $7.6 \pm 4.1$ ) were evaluated. The mean MoCA score was $19.2 \pm 4.2$, the mean MMSE score $26.2 \pm 3.2$.

In univariate analyses, we found a significant association of both MoCA and MMSE scores with age, education, gender, atrophy and DTI but not with WM hyperintensities and lacunar infarcts (table 1).

In linear regression models, FA was only related with MoCA ( $\beta$ : 0.222, $\mathrm{p}=0.032)$, while MD was associated with MoCA $(\beta$ : $-0.416, \mathrm{p}<0.001)$ and gender $(\beta: 0.213$, $\mathrm{p}=0.030)$. MTLA was associated only with age $(\beta: 0.448$, $\mathrm{p}<0.001)$. Finally, global cortical atrophy was related with MMSE $(\beta:-0.347, \mathrm{p}<0.001)$, age $(\beta$ : $0.358, \mathrm{p}<0.001)$ and gender $(\beta: 0.315, \mathrm{p}=0.001)$.

In the first model of partial correlation analysis, adjusting for demographic variables, only MoCA was significantly associated with MD while both tests were related with FA. In the second model, after removing the effect of both demographics and neuroimaging variables, we found the same significant correlations (table 2).

\section{Conclusions}

In this comparison between two widely used cognitive screening tests, when adjusting for demographics and other MRI variables, only MoCA correlated with MD, while both tests correlated with FA. We did not find any association between both cognitive tests and cerebrovascular lesions detected by conventional neuroimaging.

Perhaps, the somehow stronger association of MoCA with ultrastructural WM damage seen in our study could be considered an indirect support for the use of this screening test in patients with cognitive impairment related to SVD.

\section{Acknowledgment}

The VMCI-Tuscany study is sponsored by the Tuscany Region. 


\section{References}

1 Nasreddine ZS, Phillips NA, Bédirian V, et al: The Montreal Cognitive Assessment, MoCA: a brief screening tool for mild cognitive impairment. J Am Geriatr Soc 2005;53:695-699.

-2 Pendlebury ST, Cuthbertson FC, Welch SJ, et al: Underestimation of cognitive impairment by Mini-Mental State Examination versus the Montreal Cognitive Assessment in patients with transient ischemic attack and stroke: a population-based study. Stroke 2010;41:1290-1293.

- 3 Jokinen H, Schmidt R, Ropele S, et al; LADIS Study Group: Diffusion changes predict cognitive and functional outcome: the LADIS study. Ann Neurol 2013;73:576-583.

4 Poggesi A, Salvadori E, Pantoni L, et al: Risk and determinants of dementia in patients with mild cognitive impairment and brain subcortical vascular changes: a study of clinical, neuroimaging, and biological markers - the VMCI-Tuscany study: rationale, design, and methodology. Int J Alzheimers Dis 2012;2012:608013.

10

\section{Failure to Correct Hyperoxia Is Associated with Higher Case Fatality in Ventilated Patients with Intracerebral Hemorrhage}

\author{
F. Rincon ${ }^{a, b}$, M. Vibbert ${ }^{a}, b$, J. Urtecho ${ }^{a, b}$, M.K. Athar ${ }^{b, c}$, J. Jallo ${ }^{b}$, \\ R. Bella, b, d
}

Divisions of Critical Care and Neurotrauma, Departments of ${ }^{a}$ Neurology and ${ }^{b}$ Neurosurgery, and Departments of ${ }^{c}$ Medicine and ${ }^{\mathrm{C} C e r e b r o v a s c u l a r}$ Diseases, Thomas Jefferson University, Philadelphia, Pa., USA

\section{Background}

Intracerebral hemorrhage ( $\mathrm{ICH})$ remains the most untreatable type of stroke. Because no interventions have consistently shown to improve mortality or functional outcomes after ICH, recent basic science research, adapted from models in cerebral ischemia, have concentrated on studying the effects of normobaric hyperoxia (NBO) as a treatment modality for ICH. An animal model of $\mathrm{NBO}$ after ICH demonstrated that this therapy neither worsened nor improved outcome in animals exposed to high oxygen concentrations [1]. One of the proposed mechanisms by which NBO would be beneficial in ICH is related to the ability of $\mathrm{NBO}$ to alter the expression of matrix metalloproteinases, which are upregulated in areas surrounding the hematoma [2]. For example, a recent study demonstrated that the expression of MMP-9 could be blunted by $\mathrm{NBO}$ [3]. A major concern with NBO therapy in $\mathrm{ICH}$ may be related to its potential to aggravate the oxidative stress in the surrounding hematoma [4]. As ventilation and oxygenation remain understudied strate- gies after ICH, we sought to test the hypothesis that failure to correct hyperoxia was associated with higher inhospital case fatality in ventilated patients with $\mathrm{ICH}$ admitted to the intensive care unit (ICU).

\section{Methods}

The study was designed as a retrospective multicenter cohort study. Data were derived from Cerner Project Impact (PI) (Bel Air, Md., USA). Ventilated ICH patients were admitted within $24 \mathrm{~h}$ to the ICU at 77 United States hospitals between 2003 and 2008. To assess the effect of medical complications on in-hospital mortality, we collected data on organ dysfunction during the ICU stay according to the definitions of PI [5]. Hospitals were divided by hospital location into: urban, suburban and rural; by type into community (nonacademic), academic (university based) and public, and according to the criteria of Halpern et al. [6] into small-medium size ( $<300$ beds), large (301-499 beds) and extra large ( $>500$ beds). Specific data on the National Institutes of Health Stroke Scale, ICH score or admission imaging were not available from the PI repository. Patients were divided into three exposure groups: hyperoxia $\left(\mathrm{PaO}_{2} \geq 300 \mathrm{~mm} \mathrm{Hg}\right)$, hypoxia $\left(\mathrm{PaO}_{2}<60 \mathrm{~mm} \mathrm{Hg}\right.$ or $\mathrm{PaO}_{2} / \mathrm{FiO}_{2}$ ratio $\left.\leq 300\right)$ and normoxia (defined as no hyperoxia or hypoxia, as previously suggested by animal and observational studies) $[5,7]$. The primary outcome was in-hospital case fatality. In multivariable analysis, generalized estimating equations were used to account for potential correlations in mortality rates among patients sampled within hospital clusters. We also performed a sensitivity analysis using propensity scores to test if hyperoxia remained a significant independent predictor of in-hospital case fatality [8].

\section{Results}

The study included 1,388 ventilated $\mathrm{ICH}$ patients [ $47 \%$ $(653 / 1,388)$ female] with a mean age of 63 years (SD 15) and median Glasgow Coma Scale (GCS) score of 5 (interquartile range $3-8$ ). The overall case fatality was $59 \%$ $(812 / 1,388)$. Upon admission to the ICU, 36\% $(492 / 1,388)$ were normoxic, $47 \%(641 / 1,388)$ were hypoxic and $17 \%$ $(238 / 1,388)$ were hyperoxic. After initial resuscitation, $46 \%(352 / 780)$ were normoxic, $45 \%$ (352/780) were hypoxic and $9 \%(67 / 780)$ were hyperoxic. Of the initially admitted hyperoxic patients, $15 \%(21 / 138)$ remained hyperoxic and had a case fatality of $82 \%(18 / 21)$ compared to 
$49 \%(67 / 138)$ who became normoxic and had a case fatality of $46 \%$ (32/67; crude odds ratio, 6.6 ; $95 \%$ CI, $1.8-25$, $\left.\chi^{2}=9.4, p=0.002\right)$. In a multivariable analysis controlling for other predictors of poor outcome and hospital-specific characteristics, and a propensity score, failure to correct hyperoxia was associated with higher case fatality (adjusted odds ratio, 2.5; 95\% CI, 1.1-6.1, $\mathrm{p}=0.04$ ). Additional factors associated with higher case fatality were: non-rural hospital admission, comorbid conditions, male sex, do not resuscitate (DNR) status, GCS $<8$, mean arterial pressure $<60 \mathrm{~mm} \mathrm{Hg}$, anemia, absence of intracranial pressure monitors, organ dysfunction in the ICU and hypoxia. In our sensitivity analysis, the following variables were predictors of hyperoxia exposure in ventilated ICH patients: GCS $<8$, GCS $8-12$, admission to non-small or non-medium to large hospital size. No other variables were significantly associated with hyperoxia exposure. We did not observe significant interactions in the variables retained in the generalized estimating equation model.

\section{Conclusion}

In this large multicenter cohort study of ventilated ICH patients, we have demonstrated that failure to correct admission hyperoxia was associated with higher inhospital case fatality. The effect is robust and independent of other factors associated with poor outcome, including patient-specific factors, hospital characteristics and the probability of being exposed to hyperoxia. To our knowledge, this is the first observational study reporting the association between failure to normalize hyperoxia upon admission to the ICU and in-hospital case fatality in ventilated ICH patients, which differs from results of prior observational studies in nonmedical and stroke populations. Our data underscore the need for studies of controlled reoxygenation in ventilated ICH patients. In the absence of results from clinical trials, unnecessary oxygen delivery should be avoided in ventilated ICH patients.

\section{References}

1 Fujiwara N, Mandeville ET, Geng X, Luo Y, Arai K, Wang X, Ji X, Singhal $\mathrm{AB}$, Lo EH: Effect of normobaric oxygen therapy in a rat model of intracerebral hemorrhage. Stroke 2011;42:1469-1472.

>2 Power C, Henry S, Del Bigio MR, Larsen PH, Corbett D, Imai Y, Yong VW, Peeling J: Intracerebral hemorrhage induces macrophage activation and matrix metalloproteinases. Ann Neurol 2003;53:731-742.

-3 Sun L, Zhou W, Mueller C, Sommer C, Heiland S, Bauer AT, Marti HH, Veltkamp R: Oxygen therapy reduces secondary hemorrhage after thrombolysis in thromboembolic cerebral ischemia. J Cereb Blood Flow Metab 2010;30:1651-1660.

-4 Nakamura T, Keep RF, Hua Y, Hoff JT, Xi G: Oxidative DNA injury after experimental intracerebral hemorrhage. Brain Res 2005;1039:30-36.

5 Rincon F, Kang J, Maltenfort M, Vibbert M, Urtecho J, Athar MK, Jallo J, Pineda C, Tzeng D, McBride W, Bell R: Association between hyperoxia and mortality after stroke: a multicenter cohort study. Crit Care Med 2014;42:387-396.

6 Halpern NA, Pastores SM, Thaler HT, Greenstein RJ: Changes in critical care beds and occupancy in the United States 1985-2000: differences attributable to hospital size. Crit Care Med 2006;34:2105-2112.

7 Douzinas EE, Patsouris E, Kypriades EM, Makris DJ, Andrianakis I, Korkolopoulou P, Boursinos V, Papalois A, Sotiropoulou C, Davaris P, Roussos C: Hypoxaemic reperfusion ameliorates the histopathological changes in the pig brain after a severe global cerebral ischaemic insult. Intensive Care Med 2001;27:905-910.

8 Austin PC: An introduction to propensity score methods for reducing the effects of confounding in observational studies. Multivariate Behav Res 2011;46:399-424.

\section{1}

Inflammatory Biomarkers, Cerebral Microbleeds and Small Vessel Disease: The Framingham Heart Study

A. Shoamanesh ${ }^{a}$, S.R. Preis ${ }^{b}$, A.S. Beiser ${ }^{b, c}$, E.J. Benjamin', C.S. Kase ${ }^{c}$, P.A. Wolf', C. DeCarli' ${ }^{\text {, J.R. Romero }}$, S. Seshadric

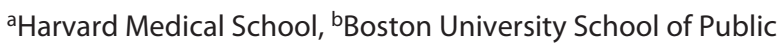
Health, and 'Boston University School of Medicine, Boston, Mass., and dUniversity of California, Davis School of Medicine, Sacramento, Calif., USA

\section{Background}

Cerebral small vessel disease (CSVD) assessed using brain magnetic resonance imaging (MRI) is characterized by three main findings that include cerebral microbleeds (CMBs), covert brain infarcts (CBIs) and white matter hyperintensities (WMHs). In recent years, there has been growing interest in alternate mechanistic causes of CSVD, including activation of the inflammatory cascade with endothelial failure and neurovascular unit dysfunction. Accordingly, we aimed to assess the association of a comprehensive panel of systemic inflammatory markers with MRI measures of CSVD.

\section{Methods}

We assessed a panel of 15 biomarkers, representing systemic inflammation [C-reactive protein, IL-6, monocyte chemotactic protein-1, TNF- $\alpha$, TNF receptor 2 (TNFR2), osteoprotegerin and fibrinogen], vascular in- 
Table 1. Logistic regression results for the association of each biomarker and prevalent CMBs $(\mathrm{n}=1,763)$

\begin{tabular}{|c|c|c|c|}
\hline \multirow[t]{2}{*}{ Biomarker } & \multicolumn{3}{|c|}{ Any CMB $(\mathrm{n}=141)$} \\
\hline & OR & $95 \% \mathrm{CI}$ & $\mathrm{p}$ value \\
\hline IL-6 (pg/ml) & 1.01 & $0.99-1.04$ & 0.29 \\
\hline ln C-reactive protein & 1.06 & $0.89-1.25$ & 0.51 \\
\hline $\ln \mathrm{TNF}-\alpha$ & 1.32 & $0.88-1.98$ & 0.18 \\
\hline ln TNFR2 & 2.17 & $1.14-4.15$ & 0.02 \\
\hline Fibrinogen (mg/dl) & 1.00 & $1.00-1.00$ & 0.52 \\
\hline Osteoprotegerin (pmol/l) & 1.06 & $0.96-1.17$ & 0.26 \\
\hline $\ln \mathrm{MCP}-1$ & 0.96 & $0.57-1.63$ & 0.89 \\
\hline In CD40 ligand & 0.95 & $0.82-1.10$ & 0.50 \\
\hline ln ICAM-1 & 1.15 & $0.57-2.30$ & 0.70 \\
\hline ln P-selectin & 1.37 & $0.82-2.28$ & 0.23 \\
\hline $\mathrm{tHcy}(\mu \mathrm{mol} / \mathrm{l})$ & 1.02 & $0.97-1.07$ & 0.47 \\
\hline ln Lp-PLA 2 activity & 0.73 & $0.34-1.60$ & 0.43 \\
\hline $\ln \mathrm{Lp}-\mathrm{PLA}_{2}$ mass & 1.10 & $0.62-1.97$ & 0.74 \\
\hline VEGF (ng/ml) & 1.00 & $1.00-1.00$ & 0.98 \\
\hline $\ln \mathrm{MPO}$ & 1.48 & $1.08-2.04$ & 0.01 \\
\hline
\end{tabular}

$\ln =$ Natural log; MCP-1 = monocyte chemotactic protein 1; tHcy = total homocysteine; VEGF = vascular endothelial growth factor. OR was adjusted for age, sex, time between blood sampling and MRI acquisition, presence or absence of CBIs and natural log transformed $\mathrm{WMH}$ volume.

Table 2. Logistic regression results for the association of each biomarker with the presence of CBIs and/or extensive WMH volume $(\mathrm{n}=1,763)$

\begin{tabular}{llll}
\hline Biomarker & \multicolumn{3}{l}{$\begin{array}{l}\text { Presence of CBI and/or extensive } \\
\text { WMH volume (n=522) }\end{array}$} \\
\cline { 2 - 4 } & OR & $95 \%$ CI & p value \\
\hline IL-6 (pg/ml) & 1.02 & $1.00-1.04$ & 0.06 \\
ln C-reactive protein & 1.01 & $0.91-1.11$ & 0.92 \\
ln TNF-a & 0.91 & $0.70-1.17$ & 0.46 \\
ln TNFR2 & 1.14 & $0.77-1.70$ & 0.51 \\
Fibrinogen (mg/dl) & 1.00 & $1.00-1.00$ & 0.96 \\
Osteoprotegerin (pmol/l) & 1.09 & $1.02-1.16$ & 0.01 \\
ln MCP-1 & 1.04 & $0.76-1.42$ & 0.80 \\
ln CD40 ligand & 1.04 & $0.95-1.13$ & 0.39 \\
ln ICAM-1 & 1.66 & $1.10-2.52$ & 0.02 \\
ln P-selectin & 1.01 & $0.76-1.35$ & 0.95 \\
tHcy $(\mu m o l / 1)$ & 1.01 & $0.98-1.04$ & 0.51 \\
ln Lp-PLA 2 activity & 1.36 & $0.86-2.15$ & 0.19 \\
ln Lp-PLA 2 mass & 1.53 & $1.09-2.14$ & 0.01 \\
VEGF (ng/ml) & 1.00 & $1.00-1.00$ & 0.41 \\
ln MPO & 0.82 & $0.68-0.99$ & 0.04 \\
\hline
\end{tabular}

$\ln =$ Natural log; MCP-1 = monocyte chemotactic protein 1 ; tHcy = total homocysteine; VEGF = vascular endothelial growth factor. OR was adjusted for age, sex, time between blood sampling and MRI acquisition, and presence of any CMB. flammation [intercellular adhesion molecule-1 (ICAM1), CD40 ligand, P-selectin, lipoprotein-associated phospholipase $\mathrm{A}_{2}$ (Lp-PLA $\mathrm{PL}_{2}$ mass and activity, total homocysteine and vascular endothelial growth factor] and oxidative stress [myeloperoxidase (MPO)] in 1,763 stroke-free Framingham offspring participants (mean age $60.2 \pm 9.1$ years, $53.7 \%$ women). MRI markers of CSVD were divided into hemorrhagic (CMBs) and ischemic (CBIs and/or extensive WMH) subtypes. Participants were determined to have extensive WMH if the natural $\log$ of the ratio of WMH volume to total cranial volume was $>1$ SD above the age-adjusted mean WMH volume. CBIs and CMBs were rated as per standard practice. Natural logarithmic transformation was performed on inflammatory biomarkers that had a skewed distribution, as well as on WMH volume to total cranial volume ratios. Multivariate logistic regression was used to obtain odds ratios (ORs) and 95\% confidence intervals (CIs) for the association of each biomarker and each outcome (CMBs and $\mathrm{CBI} /$ extensive $\mathrm{WMH}$ ). Our primary model adjusted for age, sex, time interval between baseline examination (time of blood sampling) and MRI acquisition, and the other MRI measures of interest (CMBs for the ischemic CSVD analysis, and CBIs and WMH volume for the CMB analysis).

\section{Results}

CMBs were present in $8 \%$ of the sample $(n=141)$, and $63 \%$ of cases had strictly lobar CMBs. In multivariate analysis (table 1), higher levels of circulating TNFR2 (OR 2.2, 95\% CI 1.1-4.2) and MPO (OR 1.5, 95\% CI 1.1-2.0) were each associated with CMB presence. These associations remained significant after additionally adjusting for hypertension, statin use and antithrombotic medication use. CBIs were present in $13 \%(n=222)$ of the sample and $21 \%(\mathrm{n}=370)$ were deemed to have extensive WMH volume. The combined outcome of CBI and/or extensive $\mathrm{WMH}$ volume occurred in 30\% $(\mathrm{n}=522)$. In multivariate analysis (table 2), higher levels of circulating osteoprotegerin (OR 1.1, 95\% CI 1.0-1.2), ICAM-1 (OR 1.7, 95\% CI 1.1-2.5) and Lp-PLA 2 mass (OR 1.5, 95\% CI 1.1-2.1), and lower levels of MPO (OR 0.8, 95\% CI 0.7-1.0) were associated with the presence of CBIs and/or extensive WMH volume. These associations remained significant after additionally adjusting for hypertension, diabetes mellitus, current smoking and prevalent cardiovascular disease (coronary heart disease, heart failure and peripheral vascular disease). 


\section{Conclusion}

Our study supports a possible role for inflammation in the pathogenesis of CSVD, but suggests that differing inflammatory pathways may underlie ischemic and hemorrhagic subtypes. If replicated in other samples, these biomarkers may improve stroke risk prognostication and could point to novel therapeutic targets directed at CSVD.

\section{2 \\ Left Atrial Appendage Occlusion for Stroke Prevention in Atrial Fibrillation: Multicenter Experience with the Amplatzer Cardiac Plug}

A. Tzikas ${ }^{a}$, b, S. Shakir', H. Sievert ${ }^{d}$, H. Omran ${ }^{e}$, S. Bertif, G. Santorog, J. Keferh, U. Landmesser', J.E. Nielsen-Kudsk', I. Cruz-Gonzalezk,

S. Gafoor ${ }^{d}$, T. Tichelbäcker', P. Kanagaratnam ${ }^{m}$, F. Nietlispach ${ }^{c, i}$,

A. Aminian ${ }^{n}$, F. Kasch ${ }^{\circ}$, X. Freixa ${ }^{\text {p, a , P. Danna }}{ }^{r}$, M. Rezzaghif,

P. Vermeersch' ${ }^{s}$, F. Stock ${ }^{e}$, M. Stolcova ${ }^{g}$, M. Costat ${ }^{t}$, R. Ibrahim ${ }^{q}$,

W. Schillinger', B. Meier', J.-W. Park ${ }^{\circ}$

Collaborators: W. Budts" ${ }^{u}$, T. Depotter ${ }^{v}$, E. Benitw ${ }^{w}$, F. Stammen ${ }^{x}$, S. Gloekler ${ }^{c}$, R. Eckhardt ${ }^{d}$, M. Koa-Wing ${ }^{m}$, R. Proiettir ${ }^{r}$,

M. Jaguszewski', L. Paiva ${ }^{t}$

${ }^{a}$ AHEPA University Hospital, and ${ }^{\mathrm{b}}$ Interbalkan European Medical Center, Thessaloniki, Greece; 'University Hospital of Bern, Bern, Switzerland; dCardiovascular Center Frankfurt, Frankfurt, and University Hospital of Bonn, Bonn, Germany; ${ }^{f}$ Fondazione Toscana Gabriele Monasterio, Massa, and ${ }^{9}$ Ospedale Careggi di

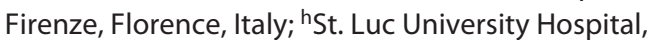
Brussels, Belgium; 'University Hospital of Zurich, Zurich, Switzerland; 'Aarhus University Hospital, Skejby, Denmark; kUniversity Hospital of Salamanca, Salamanca, Spain; 'Universitätsmedizin Göttingen, Göttingen, Germany; 'mImperial College Healthcare NHS Trust, London, UK; ${ }^{\mathrm{n}}$ Centre Hospitalier Universitaire de Charleroi, Charleroi, Belgium; ${ }^{\circ}$ Asklepios

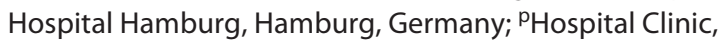
University of Barcelona, Barcelona, Spain; 'Montreal Heart Institute, Montreal, Que., Canada; 'Ospedale Luigi Sacco, Milan, Italy; ${ }^{\mathrm{s} C a r d i o v a s c u l a r}$ Institute Middelheim, Antwerp, Belgium; tCoimbra's Hospital Center, Coimbra, Portugal; "University Hospital of Leuven, Leuven, 'Cardiovascular Center Aalst, Aalst, wJessaziekenhuis, Hasselt, and XAZ Delta Hospital, Roeselare, Belgium

\section{Background/Aims}

Left atrial appendage occlusion (LAAO) is considered a valuable alternative to oral anticoagulation therapy for stroke prevention in patients with nonvalvular atrial fibrillation (AF). The Amplatzer cardiac plug is a self-ex- pandable device based on the well-established Amplatzer occluder technology and specifically designed for LAAO, which has demonstrated favorable feasibility and safety results in observational studies. This study aimed to investigate the safety, feasibility and efficacy of LAAO with the Amplatzer cardiac plug for stroke prevention in patients with AF.

\section{Methods}

Data from consecutive patients treated in 22 centers were prospectively collected, transferred in a dedicated database and retrospectively analyzed. The recommendation by the device manufacturer following LAAO was to prescribe acetylsalicylic acid (ASA) $80-100 \mathrm{mg}$ and clopidogrel $75 \mathrm{mg}$ daily for 1-3 months and then only ASA 80-100 mg for another 3 months. However, the choice and the duration of antithrombotic therapy were individualized depending on the patient history, indication for LAAO and physician preference.

\section{Results}

A total of 1,047 patients were included in the study. Their mean age was $75 \pm 8$ years; 576 patients $(55 \%)$ were $>75$ years old. Permanent AF was present in 594 patients (57\%), whereas 404 (39\%) had a history of stroke/transient ischemic attack. The mean $\mathrm{CHADS}_{2}$ score was $2.8 \pm 1.3$ and the mean $\mathrm{CHA}_{2} \mathrm{DS}_{2}$-VASc score was $4.5 \pm 1.6$. The mean HAS-BLED score was $3.1 \pm 1.2$. A score $\geq 3$ was present in 742 patients $(72 \%)$. The main indication for LAAO was previous major bleeding (47\%), followed by high risk for bleeding (35\%) and coronary stenting mandating triple anticoagulation (22\%; fig 1 ). In $16 \%$ of the patients, one of the indications was a stroke on warfarin. The composite of previous bleeding (major or minor) and high bleeding risk was $73 \%$. Procedural success was $97.3 \%$. There were 45 (4.3\%) periprocedural major adverse events: death 8 $(0.8 \%)$, stroke $9(0.9 \%)$, myocardial infarction $1(0.1 \%)$, cardiac tamponade 13 (1.2\%), major bleeding 13 (1.2\%) and device embolization needing surgery $1(0.1 \%)$. Eight of 13 major bleeding events were related to femoral artery access (not a necessary feature of LAA closure). All air embolism events were transient, without need for treatment or sequelae. All device embolizations reported as non-major adverse events where successfully snared and percutaneously removed without sequelae. Follow-up was complete in 1,001/1,019 (98.2\%) successfully implanted pa- 

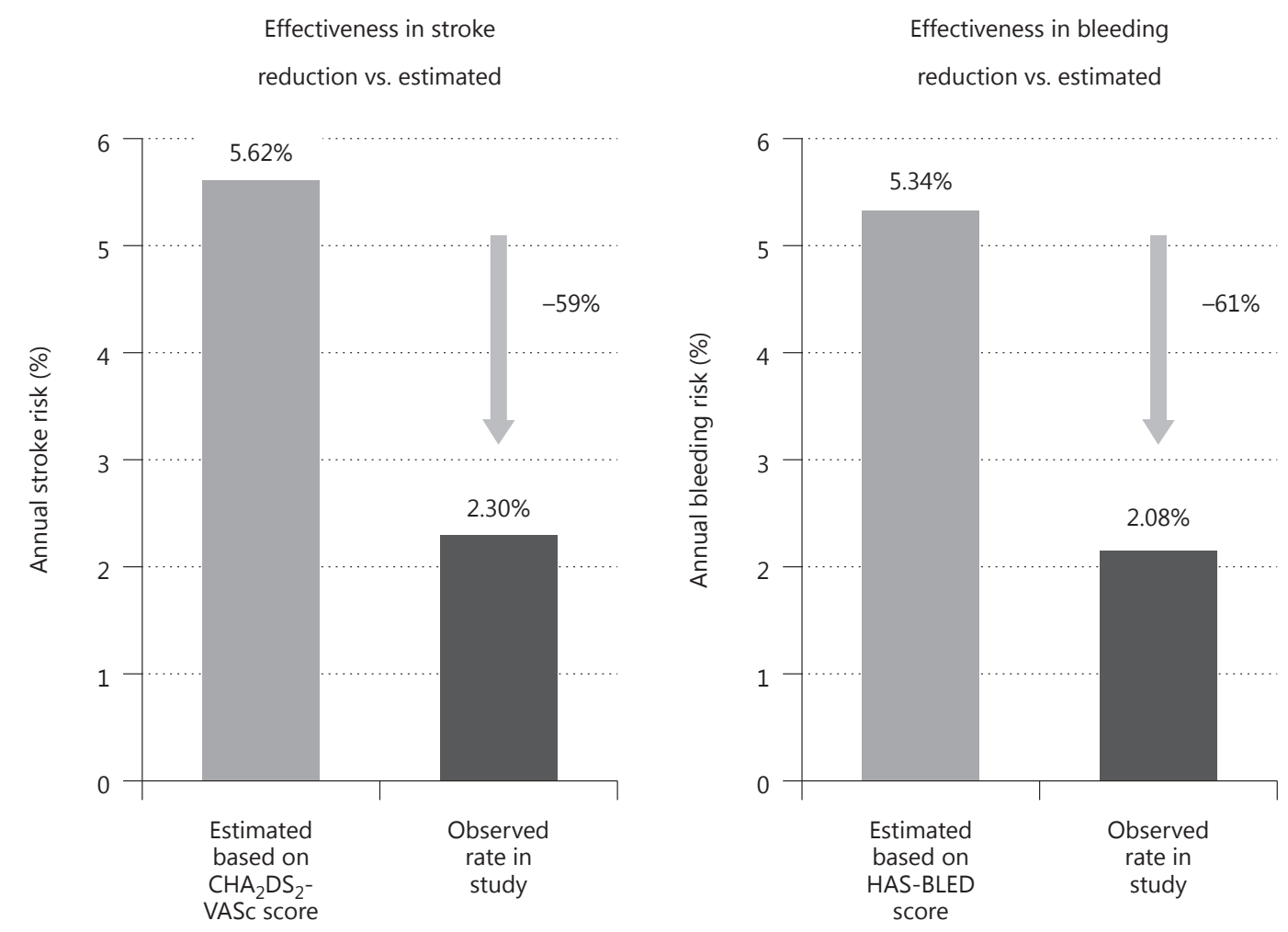
$\begin{array}{ccc}\text { Total patients } & \begin{array}{c}\text { Total patient- } \\ \text { years }\end{array} & \begin{array}{c}\mathrm{CHA}_{2} \mathrm{DS}_{2}-\mathrm{VASC} \\ \text { score }\end{array} \\ 1,001 & 1,349 & 4.43\end{array}$
Estimated stroke rate per Actual annual stroke rate
$\mathrm{CHA}_{2} \mathrm{DS}_{2}$-VASc (n strokes + TIA)
$5.62 \%$
$2.30 \%(31)$
Total patients Total patient- HAS-BLED
$\begin{array}{ccc} & \text { years } & \text { score } \\ 1,001 & 1,349 & 3.12\end{array}$

Estimated bleeding rate Actual annual bleeding rate
per HAS-BLED
( $\mathrm{n}$ major bleeds)

$5.34 \%$

$2.08 \%(28)$

Fig. 1. Indications for LAAO. TIA = Transient ischemic attack.

Fig. 2. Stroke and bleeding reduction.

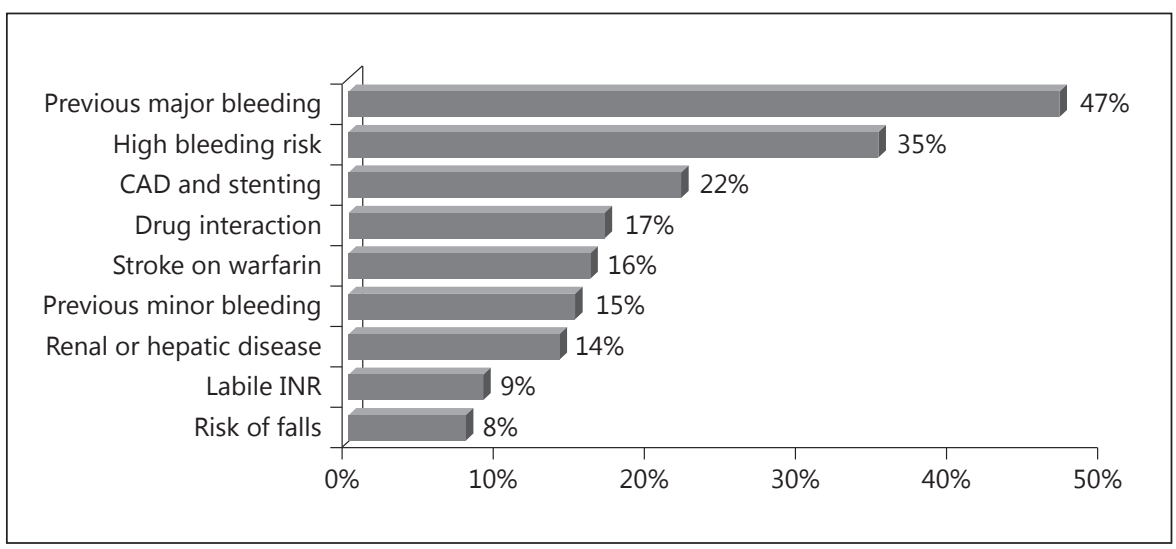
$\mathrm{CAD}=$ Coronary artery disease. 
tients (average 13 months - total 1,349 patient-years). ASA monotherapy increased from 31 to $64 \%$ and warfarin monotherapy decreased from 16 to $1.6 \%$. One-year allcause mortality was $4.2 \%$. A total of 63 deaths were reported at follow-up (17 due to cardiovascular causes). These were not reported as device related. There were $9(0.9 \%)$ strokes and $9(0.9 \%)$ transient ischemic attacks during follow-up. The annual rate of systemic thromboembolism (periprocedural and follow-up) was 2.3\% (31/1,345 patient-years), which translates into a 59\% risk reduction (fig. 2). There were 15 (1.5\%) major bleedings during follow-up. The annual rate of major bleeding (periprocedural and follow-up) was $2.1 \%$ (28/1,345 patient-years), which translates into a $61 \%$ risk reduction (fig. 2 ).

\section{Conclusions}

In this multicenter study, LAAO with the Amplatzer cardiac plug had a high procedural success and showed a favorable outcome regarding efficacy for the prevention of AF-related thromboembolism. Modification in antithrombotic therapy after LAAO resulted in the reduction of bleeding events.

\section{Disclosure Statement}

A.T., H.S., P.K., W.S., B.M. and J.-W.P are consultants/proctors and have received research grants from St. Jude Medical. H.O., S.B., G.S., J.K., J.E.N.-K., U.L., I.C.-G., F.N., A.A., X.F., P.D., M.C., R.I. and W.B. are consultants and proctors for St. Jude Medical.

\section{3 \\ Impact of Public Education (Face-Arm-Speech-Time Test) on Patient Response to Transient Ischaemic Attack and Stroke in the UK: A Population-Based Time Series Study \\ F.J. Wolters, N.L.M. Paul, L. Li, P.M. Rothwell, on behalf of the Oxford Vascular Study \\ Nuffield Department of Clinical Neurosciences, John Radcliffe Hospital, University of Oxford, Oxford, UK}

\section{Background}

Urgent medical assessment is essential after major stroke, as well as transient ischaemic attack (TIA) and minor stroke. However, fast assessment is often hindered by pre-hospital delays. The face-arm-speech-time (FAST) test was devised as a tool to increase recognition of stroke symptoms by the public, patients and paramedics, and has since formed the basis of public education campaigns in many countries, including the UK, Ireland, USA, Australia and New Zealand, with variants in several non-English-speaking countries. Despite the large international investment in the FAST campaign, no published studies have yet assessed its impact on patient response to stroke at a population level. We aimed to determine whether there has been any sustained impact of the FAST TV campaign on patient behaviour at the population level in the UK.

\section{Methods}

We prospectively determined patient behaviour after all incidences of TIA, minor stroke (National Institutes of Health Stroke Scale $\leq 3$ ) and major stroke in an ongoing UK population-based study (Oxford Vascular Study) from 2002 to 2013 . We assessed changes in patients' perception, time to seeking medical attention, hospital arrival and use of emergency medical services (EMS) before and after introduction of the national televised FAST campaign in the UK (1st April 2009), as well as per year, to assess any sustained impact of campaign continuation.

\section{Results}

Among 2,393 consecutive patients with acute cerebrovascular events, $668(27.9 \%)$ presented following major stroke, and 1,725 (72.1\%) after TIA and minor stroke. Of all major strokes, $92.6 \%$ included one or multiple FAST symptoms, whereas only $60.6 \%$ of TIA and minor strokes were FAST positive. Patients with major stroke were more likely to present directly to emergency services [odds ratio (OR) 2.18, 95\% CI 1.54-3.09, $\mathrm{p}<0.0001$ ] after the campaign and to arrive at hospital within $3 \mathrm{~h}(\mathrm{OR}=$ 2.18, 95\% CI 1.55-3.06, p < 0.0001; fig. 1). Median time (interquartile range) to seeking attention fell from 0.88 $(0.25-4.42)$ to $0.52 \mathrm{~h}(0.12-2.00 ; \mathrm{p}=0.005)$ and median time to hospital arrival from $3.08(1.47-14.75)$ to $1.98 \mathrm{~h}$ $(1.30-4.27 ; \mathrm{p}<0.0001)$. There were steep declines in the use of non-emergency services (fig. 2a) and hospital arrival within 3 h (fig. 2b) in 2009, coinciding with onset of the FAST campaign, which have been sustained thereafter. Since the campaign, patient delays have been responsible for less than one third of the delay in getting to hos- 


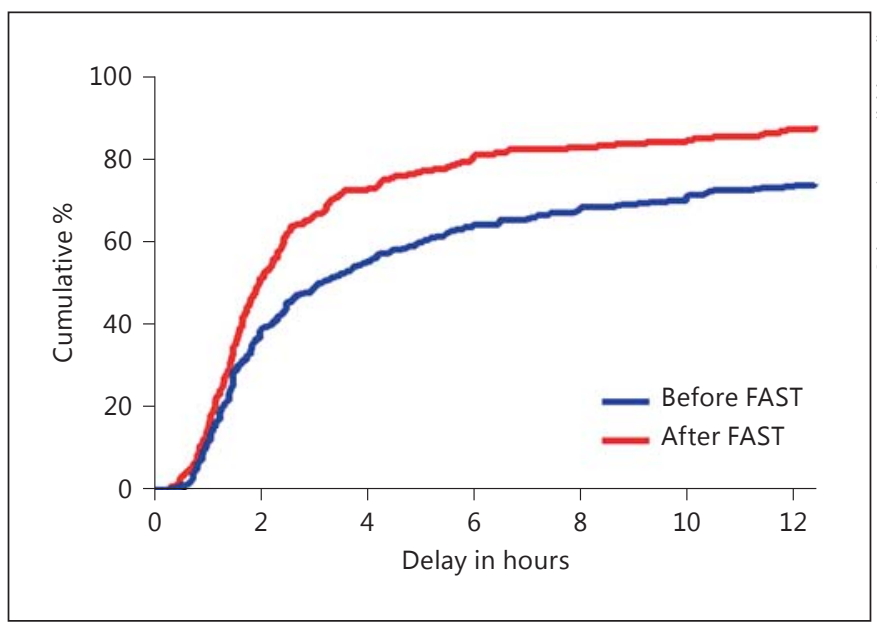

Fig. 1. Percentage of patients with major stroke that arrived in hospital by time following symptom onset. log-rank test: $\mathrm{p}<0.001$.
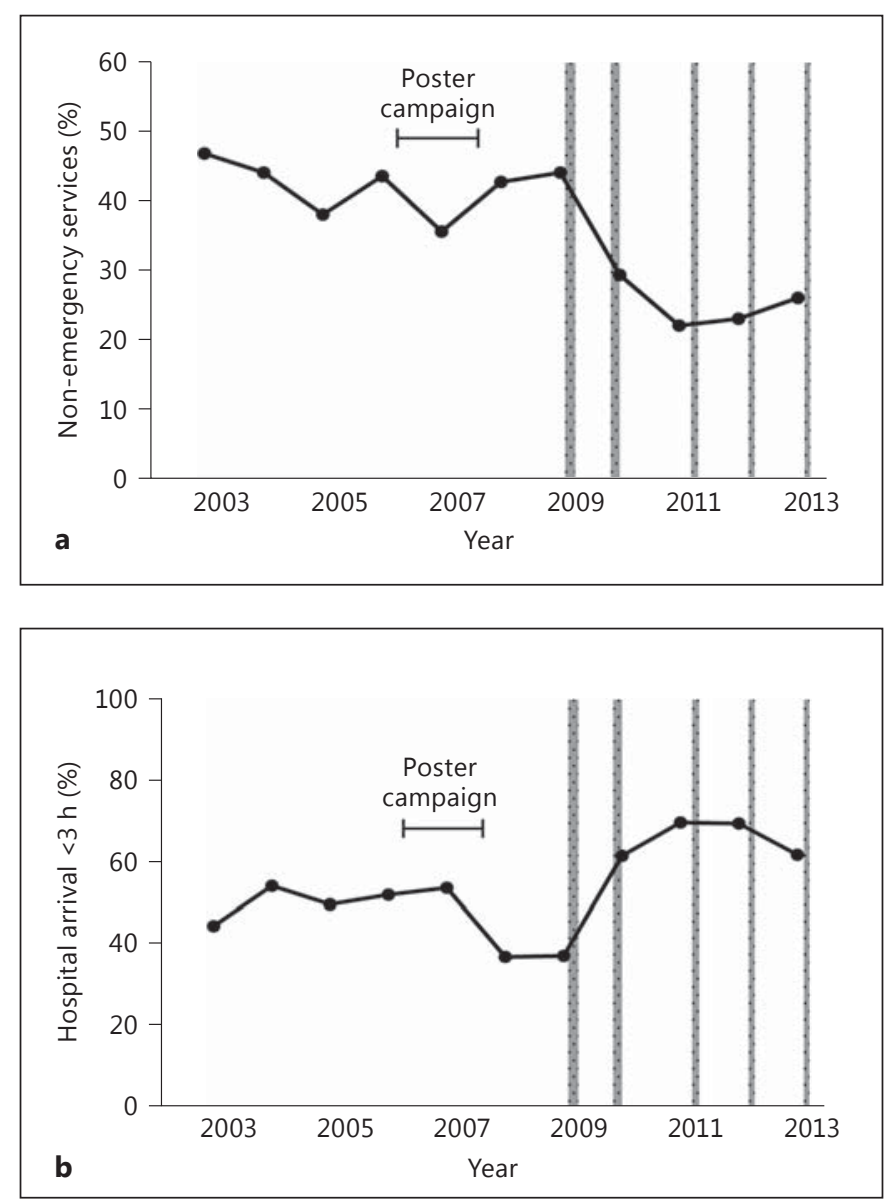

Fig. 2. The frequency (\%) after major stroke of initial non-emergency response throughout the study period (a) and hospital arrival within $3 \mathrm{~h}(\%)$ throughout the study period (b). The shaded areas reflect episodes of televised FAST campaigning. pital in those contacting EMS directly after major stroke. For TIA and minor stroke, however, there was no improvement in median time to seeking medical attention following the campaign [before $4.0(0.8-26.0)$ vs. 4.4 (0.6-37.5) after, $\mathrm{p}=0.71]$ nor in the percentage of patients seeking medical attention within $24 \mathrm{~h}$ [before $28.9 \mathrm{vs}$. $28.9 \%$ after, OR $1.00(0.79-1.26), \mathrm{p}=1.00]$. The use of EMS following TIA and minor stroke somewhat improved [OR $1.6(1.3-2.0), \mathrm{p}<0.0001$ ], but on time series analysis this did not correlate to the campaign. For TIA and minor stroke, correct perception of symptoms was strongly associated with median time (interquartile range) to seeking medical attention [correct 2.5 (10.5$18.3)$ vs. incorrect $7.0(1.0-41.0), \mathrm{p}<0.0001]$, as well as use of EMS (correct $-28.1 \%$ vs. incorrect $19.6 \%$, p = $0.0001)$. However, there was no association between perception and urgent response to symptoms for major stroke [correct $1.00(0.25-3.01)$ vs. incorrect 1.00 (0.259.53), $\mathrm{p}=0.19$; EMS $52.5 \%$ vs. non-EMS 52.4\%, OR 1.01 $(0.62-1.63), p=0.99]$. In $278(41.6 \%)$ patients with major stroke able to provide their initial perception of symptoms, $124(44.6 \%)$ correctly attributed these to stroke, which was unchanged before versus after the start of the FAST campaign [ 46.2 vs. $42.7 \%$; OR 0.87 (0.52-1.44), p = $0.58]$. In 1,438 patients with TIA and minor stroke, correct perception declined from 38.7 before to $28.4 \%$ after the campaign [OR $0.63(0.50-0.79), \mathrm{p}<0.0001]$. In the vast majority of major stroke cases $(553 / 617 ; 89.6 \%)$ and about half of TIA and minor stroke cases $(790 / 1,478$; $53.5 \%$ ), first medical attention was sought by somebody other than the patient. Details on perception of these relatives/bystanders were not collected.

\section{Conclusion}

Delays to seeking and receiving medical attention after major stroke in the UK fell strikingly in 2009, coinciding with the start of the FAST TV campaign. Campaign impact on response to TIA and minor stroke, however, was less beneficial, and symptom recognition may have been adversely affected. Future public education campaigns should continue the emphasis on urgent presentation via EMS and may benefit from additional focus on minor and transient symptoms. That medical attention was sought by a bystander after the majority of cerebrovascular events illustrates the importance of mass media public education rather than only focused programmes in high-risk groups. 


\section{Investigator-Driven Studies}

\author{
14 \\ MR Evolution of Convexal Subarachnoid \\ Hemorrhage in Cerebral Amyloid Angiopathy \\ L. Calviere ${ }^{a}$, V. Cuvinciuc ${ }^{b}$, A. Viguier ${ }^{a}$, A. Faury ${ }^{b}$, N. Raposo ${ }^{a}$, \\ C. Cognard ${ }^{b}$, V. Larrue ${ }^{a}$, F. Bonneville \\ aNeurovascular Unit, and ${ }^{\mathrm{b}}$ Department of Neuroradiology, \\ University Hospital, Toulouse, France
}

\section{Background}

Convexal subarachnoid hemorrhage $(\mathrm{cSAH})$ is increasingly recognized to be associated with cerebral amyloid angiopathy (CAA), but little is known about its evolution. We studied the initial MR features of $\mathrm{CSAH}$ in CAA and their evolution.

\section{Methods}

We performed a retrospective analysis of consecutive patients admitted to our institution for an acute nontraumatic cSAH meeting the criteria of probable or possible CAA according to the Boston criteria, who underwent initial MRI evaluation and another scanner at least once during the follow-up. The period of inclusion extended from January 2007 to January 2011. We reviewed the intensity, contrast enhancement, distribution and evolution of the sulcal and parenchymal signal abnormalities.

\section{Results}

Twenty-three patients (14 women; mean age \pm SD: $75.9 \pm 7.9$ years) were included. The most frequent clinical events were repeated transient neurological deficits including sensitive deficits with a migraine-like progression $(15 / 23 ; 65.2 \%)$ or a limb weakness $(5 / 23 ; 21.7 \%)$. Eighteen patients (78.2\%) underwent at least one followup MRI (median time 3 months; range 1-23). The mean time of follow-up \pm SD was $21.9 \pm 13.5$ months. In all patients, acute cSAH appeared as a sulcal FLAIR hyperintensity and GRE T2 hypointensity filling one or few sulci. It was combined with other hemorrhagic sequelae such as subcortical or cortical microbleeds or hematomas. The central sulcus was involved in 14 patients (60.9\%). No abnormality of the brain parenchyma facing the $\mathrm{cSAH}$ was observed. None of the patients presented with acute ischemic lesions visible on diffusion-weighted imaging. Local contrast enhancement was associated with cSAH in all but 1 of the 21 patients $(95.1 \%)$ evaluated initially by T1 with gadolinium. This contrast enhancement followed the leptomeninges in 15 patients and resembled congested vascular structures in 5 cases. In 21 patients (91.3\%), focal superficial hemosiderosis was noticed in cortical areas remote from the acute cSAH. FLAIR high-signal intensity initially observed in the sulci rapidly decreased, becoming isointense at the end of the 1st month, then hypointense. The gadolinium enhancement lasted 1-2 months and disappeared on later controls. On GRE T2, the acute bleeding left sequelae of 'railroad' superficial hemosiderosis in all patients. New subarachnoid bleedings on GRE T2 were detected in 15/18 cases (83.3\%) in distant territories. In $8 / 15$ patients, the new event was acute (hyperintense on FLAIR) or subacute (only enhanced after gadolinium). Four of these acute-subacute cSAH were symptomatic. The 7 remaining patients showed progression of superficial hemosiderosis in new cortical areas in favor of older asymptomatic cSAH already transformed into focal siderosis. Seven patients (30.4\%) presented at least one symptomatic intraparenchymal hematoma during follow-up, 2 during the 1st week and the other patients during the next months (range 1-31). Five of them had an associated cSAH adjacent to the parenchymal hematoma in the days preceding or at the time of this new acute hemorrhage.

\section{Conclusions}

In the context of CAA, cSAH is associated initially with transient contrast enhancement that may be related to pial inflammation or vascular congestion. cSAH evolves constantly into superficial hemosiderosis. This findings can allow radiologists to better determine the stage of cSAH. After the initial event, there is a high rate of $\mathrm{cSAH}$ recurrence, mostly asymptomatic, leading to progression of hemosiderosis. Moreover, patients with CAA-related cSAH seem rapidly at high risk of symptomatic intracerebral hemorrhage. Hence, acute cSAH and hemosiderosis should be considered as a warning sign for future parenchymal hematoma.

Figure 1 summarizes the initial MR features and the evolution of $\mathrm{CSAH}$ in the context of CAA. 
Fig. 1. Summary of the initial MR features and the evolution of cSAH in the context of CAA.

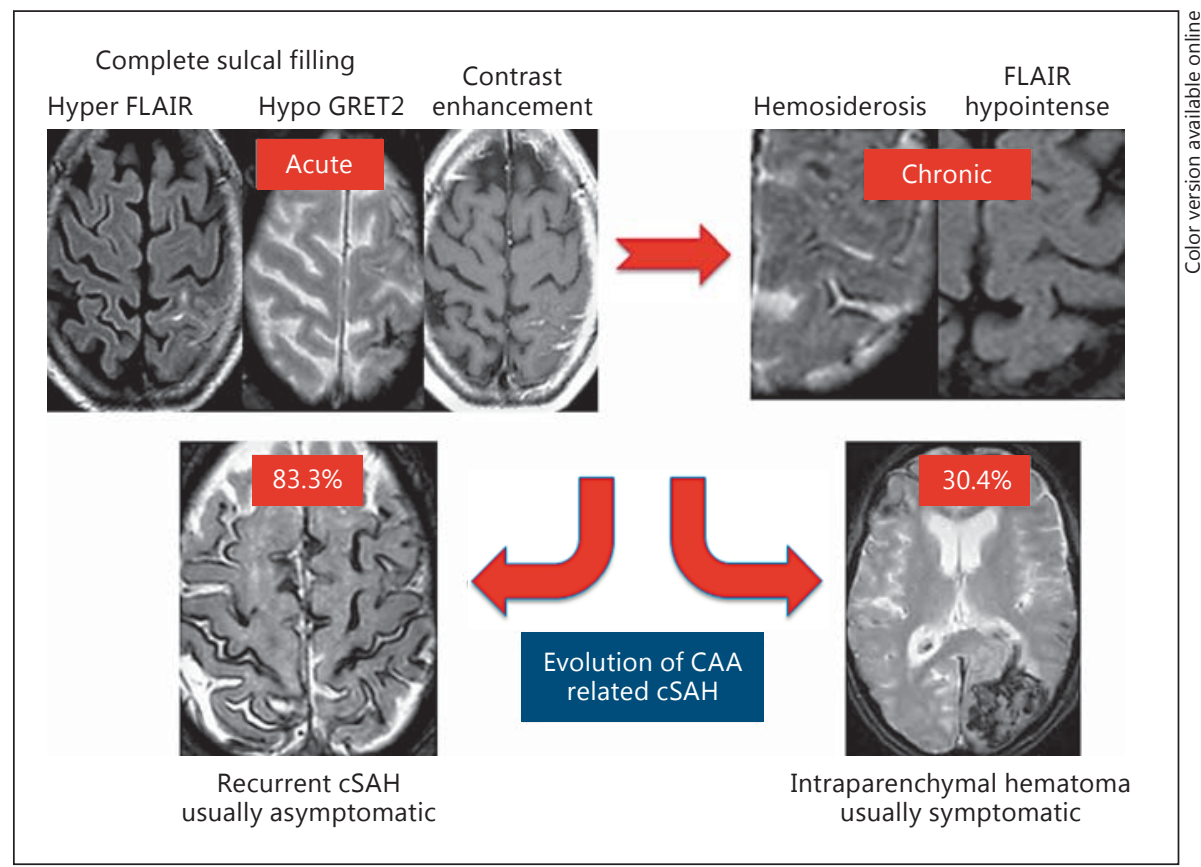

\section{5}

\section{Cerebral Microbleeds in Acute Ischemic Stroke: To Thrombolyse or Not?}

M. El-Koussy ${ }^{a}$, P.P. Gratz ${ }^{a}$, K. Hsieh ${ }^{a}$, S. von Arx ${ }^{b}$, M.-L. Mono ${ }^{b}$, M.R. Heldner ${ }^{b}$, U. Fischer ${ }^{b}$, H.P. Mattle ${ }^{b}$, M. Arnold ${ }^{b}$, C. Zubler ${ }^{a}$, G. Schroth ${ }^{a}$, J. Gralla ${ }^{a}$, S. Jung ${ }^{b}$

Departments of a Neuroradiology and ${ }^{\mathrm{b}}$ Neurology, University of Bern, Bern, Switzerland

\section{Background}

Despite continuous improvements in intravenous thrombolysis (IVT) and endovascular therapy (intra-arterial thrombolysis, IAT) for acute ischemic stroke, $7-10.5 \%$ of the patients suffer from symptomatic intracerebral hemorrhage (ICH) that deteriorates their outcome. Known risk factors for symptomatic ICH are advanced age, higher baseline National Institutes of Health Stroke Scale score, arterial hypertension, hyperglycemia and signs of early infarct demarcation on CT or MRI (table 1).

The question whether cerebral microbleeds (CMBs) visible on MRI in acute stroke increase the risk for ICH or worsen outcome after thrombolysis is unresolved. Few studies addressed this issue; however, they suffered from several methodological limitations, e.g. the location of
Table 1. Key baseline characteristics and therapy

$\begin{array}{lc}\text { Vascular risk factors } & \\ \text { Hypertension } & 63.5 \% \\ \text { Atrial fibrillation } & 46.3 \% \\ \text { Hypercholesterolemia } & 55.2 \% \\ \text { Baseline NIHSS score, median (range) } & 9(1-36) \\ \text { Occlusion localization } & \\ \text { Internal carotid artery } & 15.3 \% \\ \text { Middle cerebral artery } & 55.9 \% \\ \text { No visible occlusion } & 16.1 \% \\ \text { Previous antithrombotic therapy } & 44.4 \% \\ \text { Therapy } & \\ \text { IVT (alteplase) only } & 44.4 \% \\ \text { Bridging therapy } & 17.3 \% \\ \text { Intra-arterial urokinase } \pm \text { mechanical } & \\ \text { intervention } & 14.8 \% \\ \text { Mechanical intervention only } & 23.5 \%\end{array}$

CMBs was not taken into account or the $\mathrm{T} 2 *$ gradient echo MRI was used, which is less sensitive for CMB detection than susceptibility-weighted images (SWI). The aim of this study was to analyze the impact of CMB burden and predominant CMB locations (detected by SWI) on the bleeding risk after thrombolysis and clinical outcome in a large cohort of patients treated for acute ischemic stroke. 
Fig. 1. A 88-year-old woman presenting with dysarthria and right-sided hemiparesis (National Institutes of Health Stroke Scale 9). An area of restricted diffusion is seen in the region of the left internal capsule (a). Susceptibility-weighted imaging depicts a multitude of microbleeds of lobar distribution (b). A CT scan $24 \mathrm{~h}$ after intravenous thrombolysis (c) shows multiple hemorrhagic areas at the site of previous microbleeds. Areas of hemorrhage are all located outside of the infarcted territory as defined on diffusion-weighted imaging. The patient fulfilled the Boston criteria for probable CAA.
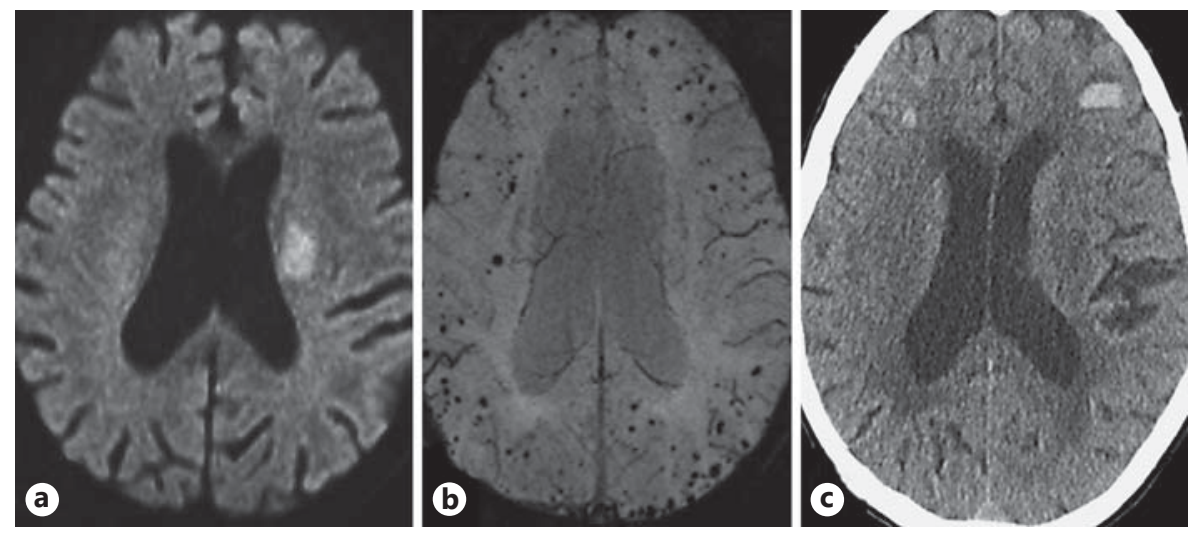

\section{Methods}

Inclusion criteria were (1) ischemic stroke diagnosed by MRI including SWI; (2) IVT, IAT or bridging therapy, and (3) follow-up MRI or CT within $72 \mathrm{~h}$ after therapy. Bleeding complications were graded according to PROACT II. From 2010 to 2013, we treated 724 patients with IVT, IAT or bridging IVT and IAT, of whom 392 patients had SWI before treatment. CMBs were rated retrospectively. The criteria to diagnose CMB on SWI were (1) round or ovoid lesion $<5 \mathrm{~mm}$; (2) area of homogeneous SWI signal loss and 'blooming', and (3) more than one half of the lesion surrounded by parenchyma (fig. 1). Multivariable regression analysis was used to determine the impact of CMBs on $\mathrm{ICH}$ and outcome.

\section{Results}

Of 392 patients (mean age \pm SD: $68.1 \pm 13.7$ years; $43.1 \%$ females), 174 were treated with IVT, 150 with IAT and 68 with bridging (table 1). CMBs were detected in 79 (20.2\%) patients; $61 / 79$ patients harbored 1 or 2 CMBs, while $10 / 79$ patients had 5 or more CMBs. No significant difference in CMB detection was noted between 1.5- $(\mathrm{n}=$ $55 ; 21.2 \%)$ vs. 3 -tesla MRI ( $\mathrm{n}=24 ; 18.0 \% ; \mathrm{p}=0.507)$. Higher age $(\mathrm{p}<0.001)$ and arterial hypertension $(\mathrm{p}=$ 0.002 ) predicted the existence of CMB in multivariable regression analysis. The presumed etiology was hypertensive encephalopathy $(36.7 \%)$, cerebral amyloid angiopathy $(26.6 \%)$ and indeterminate $(36.7 \%)$. After treatment, CT was performed in 355 patients (90.6\%) and MRI in 37 patients $(9.4 \%)$.

Symptomatic ICH occurred in $21(5.4 \%)$ and asymptomatic in 75 (19.1\%) patients; 61 (15.6\%) of these bleed- ings were within and 35 (8.9\%) outside the infarct. Neither the existence of $\mathrm{CMB}$, their burden, predominant location nor their presumed etiology influenced the ICH risk for symptomatic or asymptomatic $\mathrm{ICH}$. A higher $\mathrm{CMB}$ burden increased the risk for ICH outside the infarct slightly ( $\mathrm{p}=0.048$, odds ratio $1.004,95 \%$ CI 1.000 1.008). At the 3-month follow-up, $199 / 340$ patients (58.5\%) had a favorable outcome and 287 (84.4\%) survived. Neither outcome nor survival were associated with CMB existence $(\mathrm{p}=0.256 / \mathrm{p}=0.309)$ or burden $(\mathrm{p}=$ $0.228 / \mathrm{p}=0.412)$.

\section{Conclusion}

CMBs were detected on SWI before treatment in $20.2 \%$ (79/392) of stroke patients, which is less than the $34 \%$ previously reported in ischemic stroke. A possible explanation could be the lower number of lacunar strokes or different risk factors in our thrombolysis-treated patients compared to previous reports. The limitations of this study include the retrospective analysis and the different thrombolysis techniques used for treating our patients; however, the latter was considered in our analysis.

To conclude, CMB detected on pretreatment SWI did not increase the risk for ICH or worsen outcome, even when $\mathrm{CMB}$ burden, predominant location or presumed etiology were considered. There was only a very small increased risk for ICH outside the infarct with increasing $\mathrm{CMB}$ burden that does not advise against thrombolysis in such patients. 


\section{6}

\section{Syphilis and Cerebrovascular Disease in the 21 st Century}

F. Sousa ${ }^{a}$, S. Varanda ${ }^{a}$, J. Amorim ${ }^{b}$, M. Ribeiro ${ }^{b}$, F. Mota Garciac, J.R. Fontes ${ }^{a}$, J. Pinho ${ }^{a}$, C. Ferreira ${ }^{a}$

Departments of a Neurology, ${ }^{b}$ Neuroradiology and ${ }^{c}$ Clinical Pathology, Hospital de Braga, Braga, Portugal

\section{Background}

Syphilis is a classical example of a systemic infectious disease in which multiorganic manifestations are protean. Central nervous system involvement is thought to have been first described by Amatus Lusitanus in 1558 [1]. Mechanisms by which syphilis can cause cerebrovascular disease (CVD) include meningovascular syphilis secondary to arteritis of small, medium and large vessels, meningeal and perivascular space inflammation, and arterial dissection, namely carotid artery dissection $[2,3]$. The advent of penicillin significantly decreased chronic complications such as neurosyphilis, but more recently a significant increase in the annual incidence of syphilis was observed in developed countries $[4,5]$. Our aim was to study syphilis in a hospitalbased population of patients with CVD, and to evaluate the usefulness of routine diagnostic testing in these patients.

Table 1. Characteristics of CVD patients with neurosyphilis

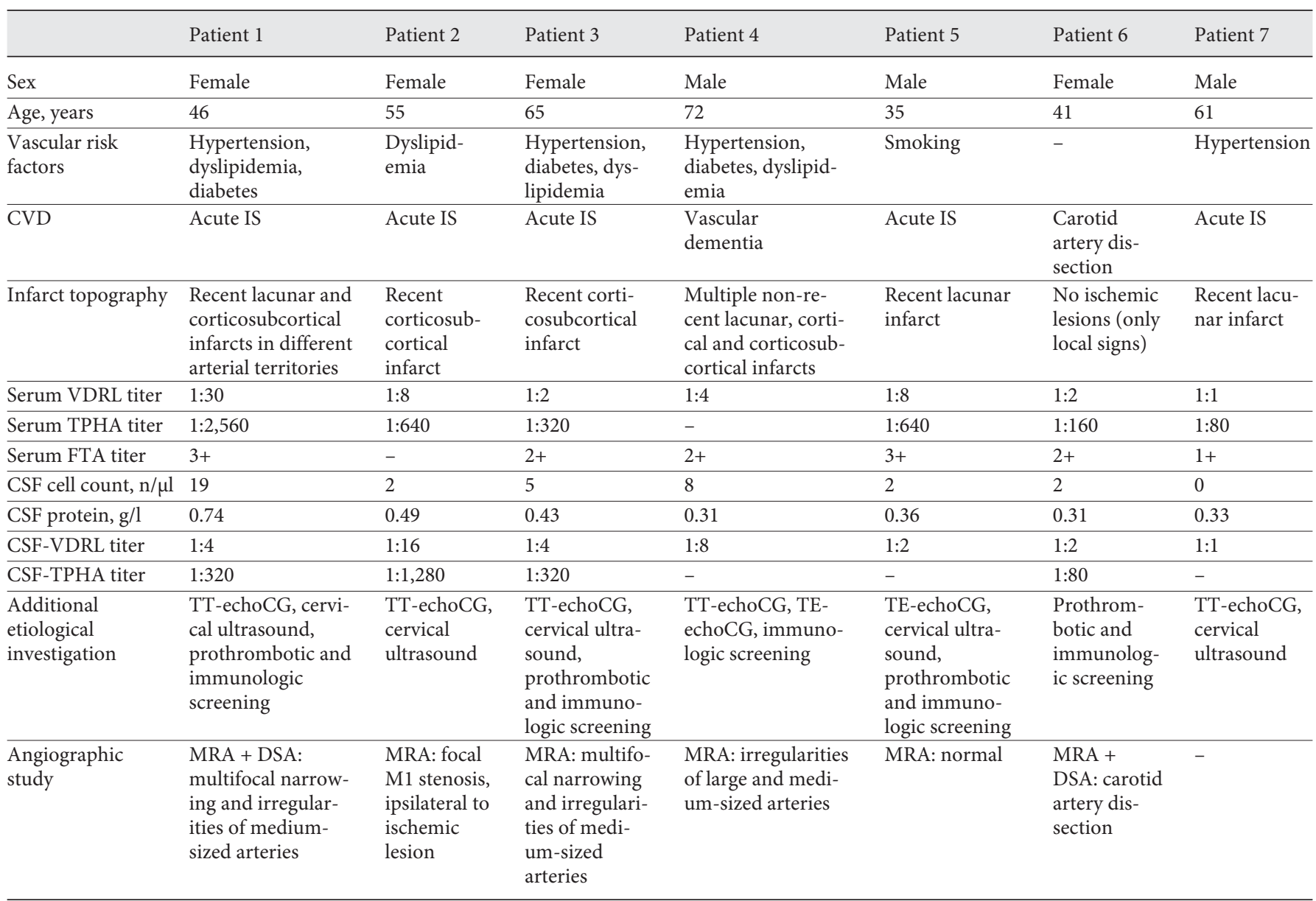

DSA = Digital subtraction angiography; FTA $=$ fluorescent treponemal antibody; TE-echoCG $=$ transesophageal echocardiogram; TPHA $=$ Treponema pallidum hemagglutination assay; TT-echoCG $=$ transthoracic echocardiogram. 


\section{Methods}

This is a retrospective study of all consecutive patients attending a tertiary university hospital, who, from December 2001 to August 2013, were tested using a blood (B-VDRL) or cerebral spinal fluid venereal disease research laboratory test (CSF-VDRL). The electronic database of the Clinical Pathology Department was searched for attached clinical information mentioning CVD, ischemic stroke (IS), transient ischemic attack (TIA), spontaneous intracranial hemorrhage and cervical artery dissection. Clinical and imaging records of adult patients with CVD, and positive B-VDRL and CSF-VDRL were individually reviewed. Cost per diagnosis of a case of systemic syphilis and a case of CVD possibly attributable to syphilis was calculated using the following formulas: VDRL test cost [6] $\times$ number of tested CVD patients/ number of CVD patients with positive B-VDRL; [(VDRL test cost $\times$ number of tested CVD patients $)+($ CSF cytochemical and microbiological test cost [6] $\times$ number of CVD patients with positive B-VDRL)]/number of CVD patients with positive CSF-VDRL.

\section{Results}

A total of 49,369 biological samples tested for BVDRL were found in the electronic database, corresponding to 34,475 patients; 2,164 patients with CVD were tested for B-VDRL, and $91.5 \%$ had acute IS or TIA; their median age was 64 years (interquartile range $=52$ 73 ) and $55.8 \%$ were male. B-VDRL was positive in 20 patients with CVD (0.9\%), and 2 additional patients had false-positive B-VDRL (positive anticardiolipin antibody). CSF-VDRL was positive in 7 CVD patients (table 1). The majority of CVD patients with positive CSFVDRL presented with acute IS $(n=5) ; 1$ patient had a non-traumatic carotid artery dissection with only local signs and 1 patient presented with acute cognitive deterioration suggestive of vascular dementia with multiple cerebral non-recent cerebral ischemic lesions. All except 1 patient had at least one of the following vascular risk factors: arterial hypertension, diabetes mellitus, dyslipidemia or smoking. All patients were HIV negative and immunocompetent. Six patients had arterial magnetic resonance angiography (MRA), and 2 of these patients underwent digital subtraction angiography. Three patients had imaging findings suggestive of vasculitis, 1 patient had cervical carotid artery dissection, 1 patient had an isolated proximal middle cerebral artery stenosis (probably atherosclerotic) and in 1 patient MRA was normal (fig. 1). CSF analysis revealed mild pleocytosis in 2 patients and mild protein elevation in 1 patient.

In our population of CVD patients, it was necessary to test B-VDRL in 108 patients to diagnose 1 case of systemic syphilis (cost per diagnosis EUR 219.65) and 309 patients to diagnose 1 case of CVD possibly attributable to syphilis (cost per diagnosis EUR 694.21).
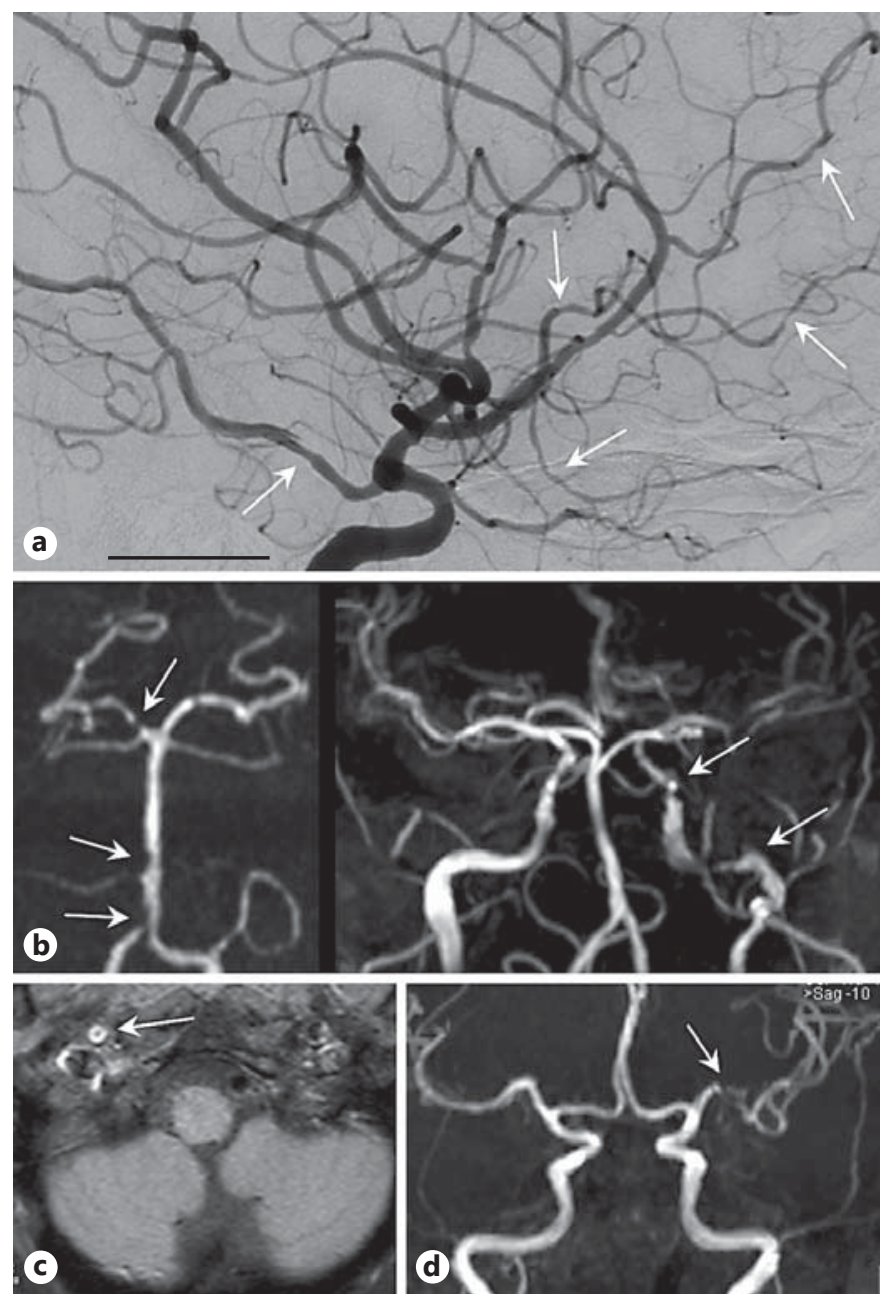

Fig. 1. a Patient 1: digital subtraction angiography showing multifocal narrowing of medium-sized intracranial arteries. b Patients 3 and 4: MR angiography showing multifocal narrowing in vertebrobasilar system arteries and a marked and longitudinally extensive irregularity in the internal carotid artery. c Patient 6: axial T1 MRI showing mural hematoma of the internal carotid artery. d Patient 2: MRA showing isolated focal M1 stenosis. 


\section{Conclusions}

Syphilis is a rare cause of CVD. In our study population, we found 7/2,164 CVD patients (0.3\%), all immunocompetent, who fulfilled the criteria for definite neurosyphilis. In 2 of these patients with neurosyphilis, we cannot be certain of the causality between treponemal infection and CVD: patient 2 had probably atherosclerotic symptomatic intracranial stenosis and patient 7 had no intracranial vascular study.

Recommendation for testing syphilis serologies in CVD patients should consider the annual incidence of local syphilis, but also the costs and benefits involved. In our center, syphilis serology is included in the first-line etiological investigation protocol for IS and TIA, but this recommendation is debatable. Our study revealed that in order to diagnose 1 case of neurosyphilis, we needed to test approximately 309 CVD patients, which, given the low cost of the VDRL test, amounted to EUR 694.21 in total. Compared to the costs and diagnostic yield of other diagnostic tests routinely performed in acute IS patients, we argue that this cost analysis per diagnosis of neurosyphilis in CVD patients is highly favorable and that a VDRL test should be recommended as first-line investigation in patients with acute IS and TIA.

This study has several limitations, e.g. its retrospective nature, the use of a clinical pathology electronic database for patient selection, absence of standardized investigation in patients with positive CSF-VDRL and the lack of follow-up data, which prevented a more comprehensive cost-effectiveness analysis.

\section{References}

1 Fontoura P: Neurological practice in the Centuriae of Amatus Lusitanus. Brain 2009;132:296-308.

2 Holmes MD, Brant-Zawadazki MM, Simon RP: Clinical features of meningovascular syphilis. Neurology 1984;34:553-556.

-3 Estol JC: Headache in cerebrovascular disease; in Caplan LR, van Gijn J (eds): Stroke Syndromes, ed 3. New York, Cambridge University Press, 2012, pp 56-57.

4 European Centre for Disease Prevention and Control: Sexually Transmitted Infections in Europe 2011. http://www.ecdc.europa.eu/en/publications/publications/sexually-transmitted-infections-europe-2011.pdf.

5 Centers for Disease Control and Prevention. Sexually Transmitted Disease Surveillance 2011. http://www.cdc.gov/std/stats.

6 Diário da República, 1ª série - No 80-24 de Abril de 2013.

\section{7}

White Matter Integrity and Depressive Symptoms in Cerebral Small Vessel Disease: The Radboud University Nijmegen Diffusion Tensor and MRI

\section{Cohort Study}

A.M. Tuladhar ${ }^{a, b}$, I.W.M. van Uden ${ }^{a}$, K.F. de Laat ${ }^{a, d}$, A.G.W. van Norden ${ }^{a, e}$, D.G. Norris ${ }^{b, f}$, g, E.J. van Dijk ${ }^{a}$, I. Tendolkar', F.-E. de Leeuw ${ }^{a}$

${ }^{\mathrm{a} D e p a r t m e n t}$ of Neurology, ${ }^{\mathrm{b} C e n t e r}$ for Cognitive Neuroimaging and 'Department of Psychiatry, Donders Institute for Brain, Cognition and Behavior, Radboud University Nijmegen Medical Center, Nijmegen, dDepartment of Neurology, Haga

Ziekenhuis, 's Gravenhage, 'Department of Neurology, Amphia Ziekenhuis, Breda, and ${ }^{\mathrm{f}} \mathrm{MIRA}$ Institute for Biomedical Technology and Technical Medicine, University of Twente, Enschede, The Netherlands; ' ${ }^{9}$ Erwin L. Hahn Institute for Magnetic Resonance Imaging, University of Duisburg-Essen, Essen, Germany

A.M. Tuladhar and I.W.M. van Uden contributed equally to this work.

\section{Background}

Depressive symptoms are common in elderly patients with cerebral small vessel disease (SVD), with a prevalence ranging from 1 to $35 \%$. SVD might cause disruption of the frontostriatal white matter (WM) tracts of the neural circuit mediating emotion perception and mood regulation, and thereby increases the risk of developing depressive symptoms in an older individual. However, as not every individual with SVD experiences depressive symptoms, other factors apart from SVD-related lesions seen on conventional MRI might be related to depressive symptoms at older age. One of these factors could be damage to the structural integrity of the normal-appearing WM (NAWM) surrounding WM hyperintensities (WMH), which cannot be assessed by conventional MRI. The microstructural organization of WM, including the NAWM, can now be assessed by diffusion tensor imaging (DTI), an MRI technique that provides valuable information on the microstructural organization of WM, including the NAWM, by measuring the molecular motion (diffusion) of water in biological tissue. We, therefore, investigated the WM integrity of WM tracts in elderly patients with depressive symptoms independent of global cognitive function by applying the tract-based spatial statistics (TBSS) on the DTI-derived parameters. 
Table 1. Baseline characteristics of the participants with and without depressive symptoms

\begin{tabular}{|c|c|c|c|c|}
\hline Characteristics & $\begin{array}{l}\text { All participants } \\
(\mathrm{n}=438)\end{array}$ & $\begin{array}{l}\text { Participants without } \\
\text { depressive symptoms } \\
(\mathrm{n}=287)\end{array}$ & $\begin{array}{l}\text { Participants with } \\
\text { depressive symptoms } \\
(\mathrm{n}=151)\end{array}$ & $\mathrm{p}$ value \\
\hline \multicolumn{5}{|c|}{ Demographic and clinical characteristics } \\
\hline Age, years (SD) & $65.1(8.8)$ & $64.8(8.9)$ & $65.6(8.6)$ & $0.377^{\mathrm{a}}$ \\
\hline Males, n (\%) & $239(54.6)$ & $169(58.9)$ & $70(46.4)$ & $0.012^{\mathrm{b}}$ \\
\hline CES-D (SD) & $11.1(9.4)$ & $5.9(4.5)$ & $21.0(8.3)$ & $<0.001^{\mathrm{a}}$ \\
\hline Antidepressive medication, n (\%) & 53 & 0 & 53 & NA \\
\hline Only primary education, $\mathrm{n}(\%)$ & $41(9.4)$ & $19(6.6)$ & $22(14.6)$ & $0.007^{\mathrm{b}}$ \\
\hline MMSE (SD) & $28.2(1.6)$ & $28.4(1.5)$ & $27.8(1.7)$ & $<0.001^{\mathrm{c}}$ \\
\hline Cognitive index (SD) & $0.00(0.45)$ & $0.04(0.43)$ & $-0.08(0.47)$ & $0.006^{\mathrm{a}}$ \\
\hline \multicolumn{5}{|l|}{ Vascular risk factors } \\
\hline Hypertension, n (\%) & $314(71.7)$ & $202(70.4)$ & $112(74.2)$ & $0.403^{\mathrm{b}}$ \\
\hline Diabetes, $\mathrm{n}(\%)$ & $60(13.7)$ & $35(12.2)$ & $25(16.6)$ & $0.207^{\mathrm{b}}$ \\
\hline Hypercholesterolemia, n (\%) & $192(43.8)$ & $118(41.1)$ & $74(49.0)$ & $0.114^{\mathrm{b}}$ \\
\hline Smoking status, n (\%) & $305(69.7)$ & $205(71.4)$ & $100(66.2)$ & $0.351^{\mathrm{b}}$ \\
\hline
\end{tabular}

Data represent numbers of participants (\%) and means (SD). MMSE = Mini-Mental State Examination; NA = not applicable. Smoking status was defined as current and former smoking. Univariate analysis: ${ }^{\mathrm{a}}$ independent $\mathrm{t}$ test, ${ }^{\mathrm{b}} \chi^{2}$ test, ${ }^{\mathrm{c}}$ Mann-Whitney $\mathrm{U}$ test.

\section{Methods}

The Radboud University Nijmegen Diffusion tensor and MRI Cohort (RUN DMC) study is a prospective cohort study in 438 older people with SVD aged 50-85 years without dementia. Depressive symptoms were assessed using the Center of Epidemiologic Studies Depression Scale (CES-D). They were considered present in patients with a CES-D score $\geq 16$ and/or current use of antidepressive medication, taken for depression, irrespective of their actual CES-D score, because depressive symptoms were considered to be the indication for the medication prescription. All patients underwent DTI. The in-house-developed algorithm named 'PATCH' was employed to the raw diffusion data to detect and correct head and cardiac motion artifacts using an iteratively reweighted least-square algorithm. Next, fractional anisotropy, mean diffusivity, axial and radial diffusivity, and mode of anisotropy images were calculated using DTIFit within the FSL toolbox, which were fed into the TBSS pipeline. Age, sex, level of education, current use of antidepressant medication, normalized total brain volume and global cognitive function were considered as possible confounders. Global cognitive function was evaluated by the Mini-Mental State Examination and the cognitive index.

\section{Results}

Demographic, clinical and neuroimaging characteristics are shown in table 1 . Mean age did not significantly differ between participants with (65.6 years, SD 8.6) or without depressive symptoms (64.8 years, SD 8.9). There were no significant differences for total WMH volume, lacunar infarcts and normalized total brain volume between the groups. Compared to nondepressed participants $(\mathrm{n}=287)$, those with depressive symptoms $(\mathrm{n}=$ 151) had lower fractional anisotropy in the genu and body of the corpus callosum, bilateral inferior fronto-occipital fasciculus, uncinate fasciculus and corona radiata. These differences disappeared after adjustment for WMH and lacunar infarcts. Mean, axial and radial diffusivity were higher in these areas in participants with depressive symptoms. After additional adjustment for WMH and lacunar infarcts, the changes observed in radial diffusivity also disappeared (fig. 1). Adding global cognition as confounding variable altered the diffusion parameters only slightly. As antidepressant medication in itself can have effects on the structural integrity of WM, we repeated the analyses while controlling for current use of antidepressant medication. These analyses did not alter our findings. 

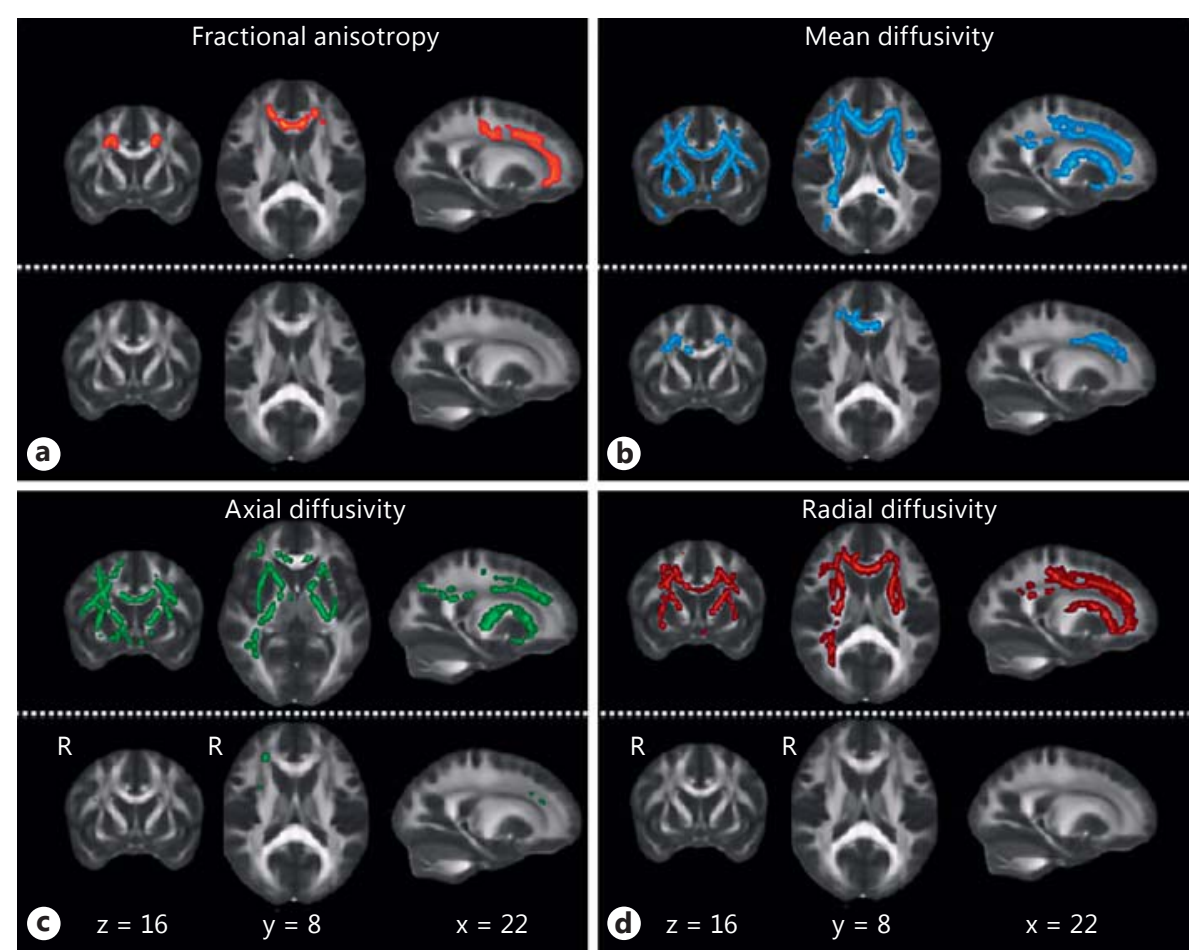

Fig. 1. Spatially distributed differences in DTI parameters between participants with and without depressive symptoms. Voxel-wise analysis of DTI parameters. a Fractional anisotropy. b Mean diffusivity. c Axial diffusivity. d Radial diffusivity. Fractional anisotropy is reduced in frontal regions in participants with depressive symptoms, while the mean, axial and radial diffusivity is increased mainly in the frontal regions $(\mathrm{p}<0.05$, family-wise error corrected for multiple comparisons). The upper panels within each box display the statistical maps of the group comparisons adjusted for age, gender, educational level and normalized total brain volume. The lower panels within each box display the statistical maps additionally adjusted for WMH volume and lacunar infarcts.

\section{Conclusions}

This study shows regional differences in the microstructural integrity of WM using TBSS in a large sample of elderly participants with or without depressive symptoms. We found that participants with depressive symptoms had a lower microstructural integrity in several $\mathrm{WM}$ regions. Lower microstructural integrity was predominantly located at the prefrontal WM fibers, disrupting the frontosubcortical circuits, which are involved in emotional processing. The loss of microstructural integrity in our participants with depressive symptoms might, at least in part, be related to the presence of SVD. However, other mechanisms might also play a role in the etiology of depression, independent of cerebrovascular disease. The analysis of the full spectrum of DTI-derived parameters is relevant and provides additional insights into the pathophysiological mechanisms that underline the occurrence of depressive symptoms in elderly patients with SVD. Both direct and indirect effects of SVD may result in axonal loss [higher axial diffusivity (AD)] and demyelinization [higher radial diffusivity (RD)]. It also helps us to refine the imaging endophenotype of patients with depressive symptoms for future studies. Future studies are, however, needed to investigate the influence of SVD not only on structural connectivity, but also on functional connectivity and the effect of treatment. This information is important in understanding the pathology of depressive symptoms in elderly patients with SVD and might be of added value to find a more specific medical treatment for elderly patients with depressive symptoms. 


\section{Meta-Analysis Review}

\section{8}

Treatment of Cerebral Cavernous Malformations: A Systematic Review and Meta-Regression Analysis

M.H.F. Poorthuis ${ }^{a}$, C.J.M. Klijn ${ }^{a},{ }^{\text {A. Algra }}{ }^{a, b}$, G.J.E. Rinkela, R. Al-Shahi Salman ${ }^{c}$

aDepartment of Neurology and Neurosurgery, Brain Center Rudolf Magnus, and bJulius Center for Health Sciences and Primary Care, University Medical Center Utrecht, Utrecht, The Netherlands; 'Division of Clinical Neurosciences, Centre for Clinical Brain Sciences, University of Edinburgh, Bramwell Dott Building, Western General Hospital, Edinburgh, UK

\section{Background}

The data on the effects of treatment from case series can help in the decision making about whether to treat a patient with a cerebral cavernous malformation (CCM) by estimating overall risks and associations with them. However, individual case series are often underpowered to do this. The aim of this study was to quantify the risk of CCM treatment more precisely and to examine study level associations with outcome after treatment.

\section{Methods}

We searched Ovid Medline, EMBASE and the Cochrane Library for peer-reviewed publications of cohort studies describing outcomes of treating 20 or more patients with a confirmed CCM diagnosis by MRI or pathology with at least $80 \%$ completeness of follow-up, and at least $90 \%$ intraparenchymal CCM. Two reviewers extracted data to quantify the incidence of a composite outcome and its constituents (death, non-fatal intracranial haemorrhage, or new or worse persistent focal neurological deficit) any time after CCM treatment. We explored associations between pre-defined summary measures of study characteristics and outcome using Poisson metaregression analyses. The characteristics of interest were average age, proportion of women, proportion of brainstem CCMs and proportion of patients presenting with CCM haemorrhage.

\section{Results}

We included 63 cohorts, involving 3,424 patients with a total of 10,029 patient-years of follow-up; 49 cohorts reported on neurosurgery in 2,684 patients with a total of 6,707 patient-years of follow-up; 14 cohorts reported on stereotactic radiosurgery in 740 patients with a total of 3,322 patient-years of follow-up. The incidence of the composite outcome was 6.1 (95\% CI 5.4-6.8) per 100 person-years of follow-up after any treatment (median follow-up 3.7 years), 6.6 (95\% CI 5.7-7.5) per 100 person-years after neurosurgical excision (median followup 3.3 years) and 5.4 (95\% CI 4.5-6.4) after stereotactic radiosurgery (median follow-up 4.0 years). The incidence of death was 0.3 (95\% CI 0.2-0.4) per 100 personyears of follow-up after any treatment, 0.3 (95\% CI $0.2-$ $0.4)$ per 100 person-years of follow-up after neurosurgical excision and 0.3 (95\% CI 0.2-0.6) per 100 person-years of follow-up after stereotactic radiosurgery. The incidence of non-fatal intracranial haemorrhage was 1.9 (95\% CI 1.6-2.2) per 100 person-years of follow-up after any treatment, 1.1 (95\% CI 0.8-1.4) per 100 person-years of follow-up after neurosurgical excision and 3.2 (95\% CI 2.7-3.9) per 100 person-years of follow-up after stereotactic radiosurgery. The incidence of a new or worse persistent focal neurological deficit was 3.2 (95\% CI 2.8-3.7) per 100 person-years of follow-up after any treatment, 4.3 (95\% CI 3.7-4.9) per 100 person-years of follow-up after neurosurgical excision and 1.2 (95\% CI 0.8-1.7) per 100 person-years of follow-up after stereotactic radiosurgery (table 1). After neurosurgical excision, the incidence of the composite outcome increased with every percent point increase in the proportion of a study of patients with brainstem CCM (risk ratio, RR, 1.03, 95\% CI 1.01$1.05)$ and decreased with each more recent study midyear (RR 0.91, 95\% CI 0.85-0.98) and each percent point increase in patients presenting with haemorrhage (RR $0.98,95 \%$ CI 0.96-1.00). We did not find significant associations in studies of stereotactic radiosurgery (table 2).

\section{Conclusion}

The reported risks of CCM treatment (and the lower risks of neurosurgical excision over time from recently bled CCMs and for CCM outside the brainstem) compare favourably with the risks of recurrent haemorrhage from CCM. The quantified risk after treatment and associations with this outcome may help to guide patient man- 
Table 1. Characteristics of the included cohorts and incidence of the composite outcome (and its constituent events) per 100 personyears of follow-up according to the modality of CCM treatment

\begin{tabular}{|c|c|c|c|c|c|c|c|c|c|}
\hline \multirow[t]{2}{*}{ Study characteristics } & \multicolumn{3}{|l|}{ Overall $(n=63)$} & \multicolumn{3}{|c|}{ Neurosurgery $(n=49)$} & \multicolumn{3}{|c|}{ Stereotactic radiosurgery $(n=14)$} \\
\hline & $\begin{array}{l}\text { number of } \\
\text { cohorts/ } \\
\text { number of } \\
\text { patients }(\%)^{\mathrm{a}}\end{array}$ & $\begin{array}{l}\text { median } \\
\text { number } \\
\text { per cohort } \\
\text { (range) }\end{array}$ & $\begin{array}{l}\text { outcome } \\
\text { event } \\
\text { incidence } \\
(95 \% \mathrm{CI})\end{array}$ & $\begin{array}{l}\text { number of } \\
\text { cohorts/ } \\
\text { number of } \\
\text { patients }(\%)^{\mathrm{a}}\end{array}$ & $\begin{array}{l}\text { median } \\
\text { number } \\
\text { per cohort } \\
\text { (range) }\end{array}$ & $\begin{array}{l}\text { outcome } \\
\text { event } \\
\text { incidence } \\
(95 \% \mathrm{CI})\end{array}$ & $\begin{array}{l}\text { number of } \\
\text { cohorts/ } \\
\text { number of } \\
\text { patients }(\%)^{\mathrm{a}}\end{array}$ & $\begin{array}{l}\text { median } \\
\text { number } \\
\text { per cohort } \\
\text { (range) }\end{array}$ & $\begin{array}{l}\text { outcome } \\
\text { event } \\
\text { incidence } \\
(95 \% \mathrm{CI})\end{array}$ \\
\hline Patients treated & $63 / 3,424(100)$ & $39(11-260)^{b}$ & & $49 / 2,684(100)$ & $39(20-260)$ & & $14 / 740(100)$ & $36(11-125)^{b}$ & \\
\hline $\begin{array}{l}\text { Duration of follow-up, } \\
\text { years }\end{array}$ & $63 / 3,424(100)$ & $2.3(0.1-8.1)$ & & $49 / 2,684(100)$ & $1.4(0.1-8.1)$ & & $14 / 740(100)$ & $4.1(0.8-6.5)$ & \\
\hline Person-years, years & $63 / 3,424(100)$ & $72(1.7-1,020)$ & & $49 / 2,684(100)$ & $48(1.7-1,020)$ & & $14 / 740(100)$ & $160(24.9-675)$ & \\
\hline Mid-year, years & $59 / 3,228(94)$ & $1996(1983-2009)$ & & $46 / 2,572(94)$ & $1997(1983-2009)$ & & $13 / 656(93)$ & $1996(1990-2001)$ & \\
\hline Age, years & $51 / 2,916(81)$ & $36(8-52)$ & & $39 / 2,323(80)$ & $35(8-52)$ & & $12 / 593(86)$ & $37(24-41)$ & \\
\hline Female, \% & $52 / 2,808(83)$ & $50(29-68)$ & & $40 / 2,215(82)$ & $50(29-68)$ & & $12 / 593(86)$ & $47(35-62)$ & \\
\hline Brainstem, \% & $56 / 3,057(89)$ & $15(0-100)$ & & $44 / 2,451(90)$ & $7(0-100)^{*}$ & & $12 / 606(86)$ & $46(2-100)^{*}$ & \\
\hline Presented with ICH, \% & $44 / 2,377(70)$ & $71(0-100)$ & & $32 / 1,825(65)$ & $47(0-100)$ & & $12 / 552(86)$ & $90(26-100)$ & \\
\hline Composite outcome & $32 / 1,568(51)$ & $3(0-108)$ & $6.1(5.4-6.8)$ & $21 / 1,100(43)$ & $2(0-108)$ & $6.6(5.7-7.5)$ & $11 / 468(79)$ & $5(1-35)$ & $5.4(4.5-6.4)$ \\
\hline $\begin{array}{l}\text { Deaths attributable to } \\
\text { CCM or treatment }\end{array}$ & $63 / 3,424(100)$ & $0(0-4)$ & $0.3(0.2-0.4)$ & $49 / 2,684(100)$ & $0(0-4)$ & $0.3(0.2-0.4)$ & $14 / 740(100)$ & $0(0-2)$ & $0.3(0.2-0.6)$ \\
\hline Symptomatic ICH & $43 / 2,465(68)$ & $1(0-32)$ & $1.9(1.6-2.2)$ & $29 / 1,725(59)$ & $1(0-22)$ & $1.1(0.8-1.4)$ & $14 / 740(100)$ & $5(0-32)$ & $3.2(2.7-3.9)$ \\
\hline Persistent FND & $42 / 2,123(67)$ & $2(0-82)$ & $3.2(2.8-3.7)$ & $31 / 1,655(63)$ & $2(0-82)$ & $4.3(3.7-4.9)$ & $11 / 468(79)$ & $2(0-6)$ & $1.2(0.8-1.7)$ \\
\hline $\begin{array}{l}\quad \mathrm{ICH}=\text { Intracranial } \\
\text { ing significant differenc } \\
\text { cohorts describing neur } \\
\text { tactic radiosurgery. }\end{array}$ & $\begin{array}{l}\text { morrhage; FN } \\
\text { in the media } \\
\text { urgical treatn }\end{array}$ & $\begin{array}{l}=\text { focal neurologic } \\
\text { roportion of this } \\
\text { t and cohorts des }\end{array}$ & $\begin{array}{l}\text { deficit; }{ }^{*} \mathrm{p}<0 . \\
\text { udy characterist } \\
\text { ibing treatment }\end{array}$ & $\begin{array}{l}5 \text {, indicat- } \\
\text { ic between } \\
\text { by stereo- }\end{array}$ & $\begin{array}{l}\text { a The percentage } \\
\text { vided by the total } n \\
{ }^{b} \text { One cohort of } 2 \\
\text { patients treated us } \\
\text { celerator. }\end{array}$ & $\begin{array}{l}\text { the number of } \\
\text { nber of cohorts } \\
\text { patients treated } \\
\text { g Gamma Knif }\end{array}$ & $\begin{array}{l}\text { cohorts reporti } \\
\text { using stereota } \\
\text { e radiosurgery }\end{array}$ & $\begin{array}{l}\text { ing on a specific st } \\
\text { ctic radiosurgery } \mathrm{W} \\
\text { and } 11 \text { patients tre }\end{array}$ & $\begin{array}{l}\text { y characteristic } \\
\text { separated into } \\
\text { ed using linear }\end{array}$ \\
\hline
\end{tabular}

Table 2. Associations between study characteristics and the incidence of the composite outcome (death, non-fatal intracranial haemorrhage, or new or worsened persistent focal neurological deficit attributed to CCM or its treatment)

\begin{tabular}{|c|c|c|c|c|c|c|c|c|c|}
\hline \multirow[t]{2}{*}{ Study characteristic } & \multicolumn{3}{|c|}{ All cohorts $(n=63)$} & \multicolumn{3}{|c|}{ Neurosurgery $(n=49)$} & \multicolumn{3}{|c|}{ Stereotactic radiosurgery $(\mathrm{n}=14)$} \\
\hline & cohorts & events & RR (95\% CI) & cohorts & events & $\mathrm{RR}(95 \% \mathrm{CI})$ & cohorts & events & RR (95\% CI) \\
\hline Mid-year, years & $*$ & & & 13 & 178 & $0.91(0.85-0.98)$ & 8 & 62 & $1.03(0.96-1.11)$ \\
\hline Age, years & 22 & 244 & $1.01(0.98-1.05)$ & 14 & 182 & $0.99(0.95-1.03)$ & 8 & 62 & $0.96(0.65-1.41)$ \\
\hline Female, $\%$ & 22 & 244 & $1.01(0.99-1.03)$ & 14 & 182 & $1.00(0.98-1.02)$ & 8 & 62 & $1.03(0.87-1.21)$ \\
\hline Brainstem, \% & 22 & 244 & $1.02(1.01-1.03)$ & 14 & 182 & $1.03(1.01-1.05)$ & 8 & 62 & $1.03(0.95-1.11)$ \\
\hline $\begin{array}{l}\text { ICH as presenting } \\
\text { symptom, } \%\end{array}$ & 22 & 244 & $1.00(0.99-1.01)$ & 14 & 182 & $0.98(0.96-1.00)$ & 8 & 62 & $1.04(0.96-1.13)$ \\
\hline
\end{tabular}

$\mathrm{ICH}=$ Intracranial haemorrhage. All analyses were adjusted for patient age, sex, proportion of brainstem CCM and proportion of patients presenting with haemorrhage, unless stated otherwise. RR are expressed per $1 \%$ increase in the proportion of patients with a study characteristic or per 1-year increase in age or mid-year. ${ }^{*} \mathrm{We}$ did not analyse the effect of mid-year on the composite outcome in all cohorts together because there was a significant interaction between mid-year and treatment modality $(\mathrm{p}=0.003)$. agement when indirectly comparing the risks of treatment and the untreated clinical course of CCM. The risks and associations we found for neurosurgical excision suggest that they compare favourably in the short term with the course of CCM outside the brainstem that have bled. The overall incidence of events after stereotactic radio- surgery was similar to neurosurgical treatment. However, long-term effects, especially important for stereotactic radiosurgery, are unknown. A randomised controlled trial is desirable to make direct comparisons of approaches to treatment where there is clinical equipoise. 